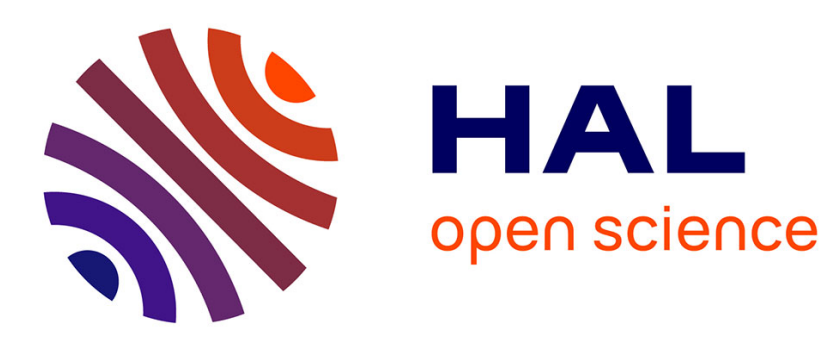

\title{
Masque indien, identité noire: l'usage politique des rituels afro-indiens par la classe ouvrière afro-américaine de La Nouvelle-Orléans
}

Aurélie Godet

\section{- To cite this version:}

Aurélie Godet. Masque indien, identité noire: l'usage politique des rituels afro-indiens par la classe ouvrière afro-américaine de La Nouvelle-Orléans. Politique Américaine, 2016, 28 (3), pp.57-87. hal02161221

\section{HAL Id: hal-02161221 \\ https://hal.science/hal-02161221}

Submitted on 20 Jun 2019

HAL is a multi-disciplinary open access archive for the deposit and dissemination of scientific research documents, whether they are published or not. The documents may come from teaching and research institutions in France or abroad, or from public or private research centers.
L'archive ouverte pluridisciplinaire HAL, est destinée au dépôt et à la diffusion de documents scientifiques de niveau recherche, publiés ou non, émanant des établissements d'enseignement et de recherche français ou étrangers, des laboratoires publics ou privés. 


\section{PDLITIQUE AMÉRICAINE}

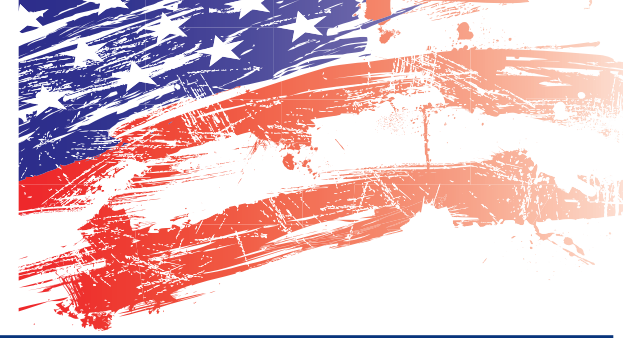

$N^{\circ} 28$ 2016

Audrey Célestine Robin D.G. Kelley Thomas Grillot Aurélie Godet David Karol Yohann Le Moigne Nicolas Martin-Breteau
La présidence Obama s'achève dans un climat de retour des fractures raciales et sociales. II aura fallu attendre l'élection du premier président noir de l'histoire du pays pour que revienne sur le devant de la scène le premier grand mouvement de lutte pour les droits des Noirs depuis les années soixante, Black Lives Matter. Ce numéro de Politique Américainey est en partie consacré, tout en analysant plus largement les dynamiques à l'œuvre dans le champ des mobilisations «par le bas » (grassroots). Les différents articles qui le composent traitent ainsi de sujets comme la saisie par les Indiens de leurs droits de pétition, la prise de conscience politique charriée par des évènements culturels comme le carnaval de La Nouvelle-Orléans, ou encore les luttes de pouvoir au niveau local entre les Noirs et les Hispaniques. Au total, Politique Américaine présente ici une réflexion sur la notion d' « empowerment » des minorités, qui reste une quête inachevée et toujours renouvelée.

\section{No \\ PロLITIQLE AMÉRICAINE}

$N^{0} 28_{12016}$

Les nouvelles formes de mobilisations raciales aux États-பnis

Black Lives Matter
et les mouvements grassroots

La recomposition locale des équilibres raciaux

Linfra-politique : l'exemple du carnaval de La Nouvelle-Orléans 


\title{
POLITIQUE \\ AMÉRICAINE
}

\author{
Directeurs de la publication \\ et rédacteurs en chef
}

ALEXANDRA DE HOOP SCHEFFER

FRANÇOIS VERGNIOLLE DE CHANTAL

\section{COMITÉ DE RÉDACTION}

- Daniel Béland, Université de la Saskatchewan - Anne-Lorraine Bujon de L'estang, revue Esprit - Frédérick Douzet, Université de Paris VIII, - Denis Lacorne, Centre d'études et de recherches internationales (CERI Sciences-Po), - Alix Meyer, Université de Bourgogne - Vincent Michelot, Institut d'études politiques de Lyon • Julien Za Rifian, Université de Cergy-Pontoise

\section{CONSEIL SCIENTIFIQUE}

Akhil Reed AMAR, professeur de droit et de science politique, Faculté de droit, Université Yale

Suzanne BERGER, professeur de science politique, M.I.T., Massachusetts Institute of Technology

Bruce CAIN, professeur de science politique, Université Stanford, Californie

James W. CEASER, professeur de science politique, Université de Virginie

Jack CITRIN, professeur de science politique, Institute for Governmental Studies, Université de Californie, Berkeley

John D. DONAHUE, professeur de science politique, John F. Kennedy School of Government, Université Harvard

Francis FUKUYAMA, professeur de relations internationales, School of Advanced International Studies, Université Johns Hopkins

Charles O. JONES, professeur émérite de science politique, Université de Wisconsin-Madison

Robert O. KEOHANE, professeur de relations internationales, Université Duke

Charles A. KUPCHAN, professeur de relations internationales, Université Georgetown

David R. MAYHEW, professeur de science politique, Université Yale

Sidney MILKIS, professeur de science politique, Miller Center, Université de Virginie
Joseph S. NYE, professeur de relations internationales, John F. Kennedy School of Government, Université Harvard

Robert B. REICH, professeur d'économie politique, Université de Californie, Berkeley

Andrew RUDALEVIGE, professeur de science politique, Bowdoin College

Larry J. SABATO, professeur de science politique, Université de Virginie

Saskia SASSEN, professeur de sociologie, Université de Columbia

Steven SKOWRONEK, professeur de science politique, Université Yale

Anne-Marie SLAUGHTER, professeur de relations internationales, Woodrow Wilson School of Public and International Affairs, Université Princeton

Rogers SMITH, professeur de science politique, Université de Pennsylvanie

Ezra SULEIMAN, professeur de science politique, Université Princeton

Serge SUR, professeur des universités, Université de Paris-Panthéon-Assas

Bruno TERTRAIS, maître de recherches, Fondation pour la Recherche Stratégique

Margaret WEIR, professeur de science politique et de sociologie, Université de Californie, Berkeley. 
Maquette réalisée par ATA AYATI

Revue publiée en partenariat avec l'Institut des Amériques et avec le soutien du Centre National du Livre et du German Marshall Fund

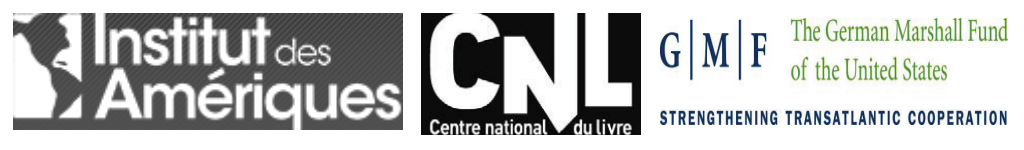

(C) L'Harmattan, 2016

5-7, rue de l'École Polytechnique ; 75005 Paris

http://www.editions-harmattan.fr

diffusion.harmattan@wanadoo.fr

ISBN : 978-2-343-10268-9

EAN : 9782343102689 


\section{Sommaire}

Dossier dirigé par Audrey Célestine et Nicolas Martin-Breteau

Éditorial

Alexandra de Hoop Scheffer et François Vergniolle de Chantal

Introduction

Audrey Célestine et Nicolas Martin-Breteau

"Un mouvement, pas un moment ": Black Lives Matter

et la reconfiguration des luttes minoritaires à l'ère Obama

Audrey Célestine et Nicolas Martin-Breteau

Frontières du politique : la pratique pétitionnaire

sur une réserve indienne (1880-1980)

Thomas Grillot

Masque indien, identité noire : l'usage politique des rituels afro-indiens par la classe ouvrière afro-américaine de La Nouvelle-Orléans

Aurélie Godet

Race, ethnicité et concurrence interminorités : le cas des rivalités politiques entre Africains-Américains et Latinos dans la ville de Compton (Californie)

YohANn Le MOIGne

Entretien avec Robin D. G. Kelley : penser la continuité des luttes pour l'égalité raciale

Réalisé par Nicolas Martin-Breteau

\section{VARIA}

Un système sous pression : Les partis politiques et l'investiture présidentielle aux États-Unis en 2016

David Karol

Compte-rendu d'ouvrages 



\title{
Masque indien, identité noire : l'usage politique des rituels afro-indiens par la classe ouvrière afro-américaine de La Nouvelle-Orléans
}

\author{
Aurélie Godet ${ }^{*}$
}

Résumé

Cet article se propose d'étudier l'héritage du binarisme racial à La Nouvelle-Orléans au travers des parades festives des Mardi Gras Indians, rituel folklorique spécifique à la ville, même si on en trouve des échos dans l'aire caraïbe et sud-américaine. Prenant acte de la longue histoire du " playing Indian» (Philip Deloria) aux États-Unis, il envisage dans un premier temps les processions organisées par ces tribus fictives $d^{\prime}$ "Indiens noirs " comme une porte dérobée vers un ailleurs racial, comme l'« invention d'une tradition» (Eric Hobsbawm) et comme l'exercice du "droit à être un autre " (Mikhaïl Bakhtine). Ces mêmes défilés, ainsi que les six à neuf mois de préparatifs qui les précèdent, sont ensuite réinterprétés comme l'expression " infra-politique » (James C. Scott) d'une identité noire conquérante et autonome, qui se rit des règles instaurées par l'élite politique et économique blanche de La Nouvelle-Orléans dans le but de canaliser l'esprit festif et marginaliser les pratiques culturelles populaires afro-américaines. Enfin, cet article revient sur la trêve conclue en 2011 entre la municipalité blanche de La Nouvelle-Orléans et les Mardi Gras Indians afin d'en démêler les implications en termes de relations de pouvoir depuis le passage de I'ouragan Katrina.

Depuis les années 1980, la science politique a considérablement élargi sa conception des répertoires et territoires de l'action politique, jusqu'à considérer parfois que «tout est politique ", pour reprendre un slogan bien connu ${ }^{1}$. Traquant les manifestations du politique dans des lieux plus marginaux que ceux

* Ancienne élève de l'École Normale Supérieure de Lyon, titulaire de l'agrégation d'anglais et d'un doctorat d'études anglophones, Aurélie Godet est depuis 2013 maître de conférences à I'Université Paris Diderot - Paris 7, où elle enseigne la civilisation américaine aux niveaux licence et master. Après avoir travaillé pendant sept ans sur l'évolution des idées conservatrices depuis les années 1930, ses recherches portent à présent sur l'histoire politique et culturelle de la Louisiane. Elle a en projet la publication d'une monographie sur le carnaval de La Nouvelle-Orléans.

1 Je remercie vivement les évaluateurs anonymes de la revue Politique américaine ainsi que les coordinateurs du numéro, Audrey Célestine et Nicolas Martin-Breteau, pour la relecture attentive qu'ils ont faite de ce texte. 
habituellement retenus (autrement dit, en dehors du champ électoral, partisan ou gouvernemental), des chercheurs aussi différents que Denis-Constant Martin en France, Alberto Melucci en Italie et James C. Scott aux États-Unis ont ainsi mis en lumière la diversité des modes d'inscription du politique ${ }^{2}$.

Dans la liste des « objets politiques non identifiés » (OPNI) que dresse cette littérature du décentrement, le sport (le football et la boxe notamment), la musique (le rock, la chanson populaire, le rap et les « musiques du monde » par exemple), la littérature, la cuisine et le vêtement tiennent sans conteste le haut du pavé . Il faudrait y ajouter, à l'initiative de James C. Scott et Denis-Constant Martin, les pratiques festives, et notamment le carnaval ${ }^{4}$. Espace-temps ritualisé, le carnaval a, en effet, pour particularité de mettre en scène publiquement, tout en les travestissant, les identités et les rapports sociaux. Ni soupape de sécurité instrumentalisée par les élites, ni création unilatérale du peuple, il contourne les modes de représentation et d'objectification dominants et signale les limites des

Sur la réticence, puis la prise en compte graduelle, des formes d'engagement politique non conformes à l'idéal démocratique de la participation, lire Arnaud, Lionel et Christine Guionnet, dir., Les Frontières du politique : enquêtes sur les processus de politisation et dépolitisation, Rennes, PUR, 2005 ; McFalls, Laurent, Julie Perreault, Nicolas Liorzou et Anca-Elena Mot, Construire le politique : contingence, causalité et connaissance dans la science politique contemporaine, Montréal, Presses de l'Université Laval, 2006.

2 Martin, Denis-Constant, Sur la piste des OPNI (objets politiques non identifiés), Paris, Karthala, 2002 ; Melucci, Alberto, Challenging Codes : Collective Action in the Information Age, Cambridge, Cambridge University Press, 1996 ; Scott, James C., Domination and the Arts of Resistance. Hidden Transcripts, New Haven, Yale University Press, 1990. Traduit en français par Olivier Ruchet sous le titre La Domination ou les arts de la résistance. Fragments du discours subalterne, Paris, Éditions Amsterdam, 2008. Voir également le numéro spécial de la revue Espaces-Temps coordonné par Sophie Duchesne et Florence Haegel ("Repérages du politiques », Espaces-Temps vol. 76-77, 2001) ainsi que celui de la Revue française d'études américaines coordonné par Guillaume Marche ("Infrapolitics and Mobilizations ", Revue française d'études américaines 131, 2012).

3 Sur le sport, voir Saavedra, Martha, Les terrains politiques du football, Paris, Khartala, 2010 ; Beauchez, Jérôme, L'empreinte du poing : la boxe, le gymnase et leurs hommes, Paris, Éditions de l'EHESS, 2014, coll. " Cas de figure »; Carrington, Ben, Race, Sport and Politics : The Sporting Black Diaspora, London, Sage, 2010. Sur la musique et la littérature, on consultera Kelley, Robin D. G., Race Rebels : Culture, Politics, and the Black Working Class, New York, Free Press, 1994, chapitre 8 ; Martin, Denis-Constant Martin, "Les 'musiques du monde' : imaginaires contradictoires de la globalisation", in Sur la piste des OPNI, p. 398-430 ; Moreiras, Alberto, "Infrapolitical Literature. Hispanism and the Border », CR: The New Centennial Review vol. 10 $n^{\circ} 2$, 2010, p. 183-204. Sur la cuisine, on lira avec profit Coulon, Christian, "La cuisine comme objet politique ", Revue internationale de politique comparée vol. 6 n², été 1999, p. 311-320 ; Le Dantec-Lowry, Hélène, "Reading Women's Lives in Cookbooks and Other Culinary Writings : A Critical Essay ", Revue française d'études américaines n¹16, 2008, p. 99-122. Sur le vêtement, enfin, on se réfèrera à Alvarez, Luis, The Power of the Zoot: Youth Culture and Resistance during World War II, Berkeley, University of California, 2008 ; Miller, Monica, Slaves to Fashion : Black Dandyism and the Styling of Black Diaspora Identity, Durham, Duke University Press, 2009 ; White, Sophie, Wild Frenchmen and Frenchified Indians : Material Culture and Race in Colonial Louisiana, Philadelphie, University of Pennsylvania Press, 2012.

4 Scott, James C., ibid, p. 172-182, 188 ; Martin, Denis-Constant, Politics Behind the Mask : Studying Contemporary Carnivals in Political Perspective. Theoretical and Methodological Suggestions, Paris, CERI, "Questions de recherche », 2001. Disponible en ligne : www.ceri-sciences-po.org/ publica/qdr.htm. Consulté le 9 mars 2016. 
oppositions binaires, ce qui a pu faire dire à Abner Cohen qu'il n'est finalement rien d'autre que «la politique avançant sous le masque de la culture $»^{5}$.

À La Nouvelle-Orléans comme dans d'autres grandes villes du Sud des ÉtatsUnis (Charleston, Savannah), ce que l'on appelle " politique » (autrement dit, les interactions entre individus au sein d'un groupe plus large dans une lutte pour le pouvoir ou la reconnaissance) met prioritairement en jeu les identités raciales. L'« étasunification » de la société et de l'espace urbain à partir du transfert de souveraineté de 1803, et encore plus à partir de la fin de la guerre de Sécession, a simplifié l'ordre racial qui prévalait depuis le XviII siècle et progressivement effacé l'urbanité métissée (mais néanmoins racialisée ${ }^{6}$ ) des périodes française et espagnole. La petite bourgeoisie de " gens de couleur libres » (affranchis ou descendants d'affranchis, issus d'unions entre propriétaires fonciers français et esclaves africaines) y a de fait perdu son statut distinct ${ }^{7}$, et la philosophie politico-raciale nord-américaine a favorisé une vision de la société « en noir et blanc ».

Le débat qui s'est dessiné pendant la Reconstruction sur la définition même du terme « créole » a confirmé cette racialisation. Jusqu’à la guerre de Sécession, le mot avait été employé à La Nouvelle-Orléans pour distinguer les natifs de la ville des résidents européens, africains ou asiatiques plus récemment arrivés, puis pour distinguer ces mêmes natifs des colonisateurs américains ${ }^{8}$. Cette identité spatiale s'est transformée en identité raciale à la fin du XIX siècle, quand les descendants blancs (ou qui se considéraient comme tels) de colons français et espagnols ont tenté de s'approprier l'exclusivité de la dénomination «créole » pour

5 Cohen, Abner, Masquerade Politics : Explorations in the Structure of Urban Cultural Movements, Berkeley, University of California Press, 1993, chapitre 10 : «The Aestheticisation of Politics ».

6 Sur la co-présence du métissage et des rapports de domination raciale au sein de la société coloniale néo-orléanaise, lire notamment Aubert, Guillaume, "The Blood of France : Race and Purity of Blood in the French Atlantic World ", William and Mary Quarterly vol. 61 n³, 2004, p. 439-478; Ingersoll, Thomas N., Mammon and Manon in Early New Orleans : The First Slave Society in the Deep Society, 1718-1819, Knoxville, University of Tennessee Press, 1999 ; Vidal, Cécile, "Caribbean Louisiana : Church, Métissage, and the Language of Race in the Mississippi Colony during the French Period ", in Louisiana : Crossroads of the Atlantic World, Philadelphia, University of Pennsylvania Press, 2013, p. 125-146 ; Williams, Mary, "Private Lives and Public Orders : Regulating Sex, Marriage, and Legitimacy in Spanish Colonial Louisiana ", in Louisiana : Crossroads of the Atlantic World, Philadelphia, University of Pennsylvania Press, 2013, p. 147-164.

7 Sur l'histoire de la population libre de couleur de La Nouvelle-Orléans dans sa diversité, lire Clark, Emily, The Strange History of the American Quadroon : Free Women of Color in the Revolutionary Atlantic World, Chapel Hill, University of North Carolina Press, 2013 ; Dessens, Nathalie, From Saint-Domingue to New Orleans : Migration and Influences, Gainesville, University Press of Florida, 2007 ; Gerhman, Mary, The Free People of Color of New Orleans : An Introduction, New Orleans, 1994 ; Hanger, Kimberly S., Bounded Lives, Bounded Places : Free Black Society in Colonial New Orleans, 1769-1803, Durham, Duke University Press, 1997 ; Kelleher Schafer, Judith, Becoming Free, Remaining Free: Manumission and Enslavement in New Orleans, 18461862, Baton Rouge, Louisiana State University, 2003 ; Spear, Jennifer M., Race, Sex, and Social Order in Early New Orleans, Baltimore, Johns Hopkins University Press, 2009.

8 Cf. Tregle, Jr., Joseph, "Creoles and Americans ", in Creole New Orleans : Race and Americanization, Hirsch, Arnold R. et Joseph Logdson, dir., Baton Rouge, Louisiana State University Press, 1992, p. 131-185. 
se différencier des «Créoles de couleur» (appellation revendiquée par les gens de couleur libres après la guerre de Sécession) ${ }^{9}$. Lapplication des lois Jim Crow, qui ont défini les règles de la ségrégation dans les espaces publics nord-américains et le principe d'un statut " séparé mais égal » pour les Noirs américains, a accentué la binarisation de la société locale dans la mesure où était considérée comme « personne de couleur» toute personne ayant $1 / 32^{\mathrm{e}}$ ou plus de sang noir ${ }^{10}$.

Depuis les années 1970 (sous l'influence du mouvement littéraire et politique de la créolité), et encore plus depuis le passage de l'ouragan Katrina, la municipalité de La Nouvelle-Orléans tente d'encourager une créolité œcuménique, à vocation essentiellement touristique et largement fondée sur l'héritage culinaire, architectural, linguistique et musical de la ville ${ }^{11}$. Mais l'héritage du binarisme est lourd, et colore encore aujourd'hui les discours sur la ville, que ce soit celui des planificateurs urbains ou celui des médias ${ }^{12}$.

9 Les Louisianais blancs d'ascendance française et/ou espagnole n'ont eu de cesse de faire reconnaître leur pureté raciale dans les années 1880, ainsi qu'en témoignent les échanges enflammés entre le romancier orléanais George Washington Cable et l'historien " créole blanc » Charles Gayarré par livres interposés. Voir à ce sujet Dominguez, Virginia, White By Definition : Social Classification in Creole Louisiana, New Brunswick, Rutgers University Press, 1986 ; Fertel, Rien, Imagining the Creole City : The Rise of Literary Culture in Nineteenth-Century New Orleans, Baton Rouge, Louisiana State University Press, 2014 ; Weil, François, in Empires of the Imagination : Transatlantic Histories of the Louisiana Purchase, Kastor, Peter J. et François Weil, dir. Charlottesville, University of Virginia Press, 2009, p. 301-326.

10 Cf. Lodgson, Joseph et Caryn Cossé Bell, "The Americanization of Black New Orleans », in Creole New Orleans : Race and Americanization, p. 201-261; Le Menestrel, Sara, La Voie des Cadiens : tourisme et identité en Louisiane, Paris, Belin, 1999, p. 100.

11 Mark Souther qualifie ce phénomène de «Disneyfication ». Souther, Mark, "The Disneyfication of New Orleans : The French Quarter as Facade in a Divided City ", Journal of American History vol. 94 n³, 2007, p. 804-811. Sur les usages contemporains du mot "créole », lire également Hirsch, Arnold, "Simply a Matter of Black and White : The Transformations of Race and Politics in Twentieth-Century New Orleans ", in Creole New Orleans : Race and Americanization, p. 262-320 ; Le Menestrel, Sara, "Créolisation, imaginaire racial et marché musical francolouisianais ", in Raulin, Anne et Susan Rogers, dir., Parallaxes transatlantiques : pour une anthropologie réciproque France-États-Unis, Paris, CNRS Éditions, 2012, p. 79-108.

12 Comme l'a souligné Arnold Hirsch en 2007, le discours sur la reconstruction de La NouvelleOrléans après 2005 a largement effacé, non seulement la population noire du Ninth Ward, mais également la population afro-créole et son empreinte sur la ville (dans les quartiers de Pontchartrain Park, New Orleans East, Tremé ou Seventh Ward, notamment). Cf. Hirsch, Arnold R., "Fade To Black : Hurricane Katrina and the Disappearance of Creole New Orleans ", Journal of American History vol. 94 n³, 2007, p. 752-761. Sur les reconstructions fantasmées de La Nouvelle-Orléans après Katrina, voir également Dyson, Michael E., Come Hell or High Water : Hurricane Katrina and the Color of Disaster, New York, Basic Civitas, 2006 ; Hernandez, Julie, "Réécritures urbaines : héritages créoles et empreinte étasunienne à La Nouvelle-Orléans ", RITA n¹, décembre 2008 ; Rydin, Yvonne, "Justice and the Geography of Hurricane Katrina ", Geoforum n³7, 2006 ; Thomas, Lynell L., Desire and Disaster in New Orleans : Tourism, Race, and Historical Memory, Durham, Duke University Press, 2014 ; Sparks, Randy J. et Romain Huret, dir., Hurricane Katrina in Transatlantic Perspective, Baton Rouge, Louisiana State University, 2014 ; Wagner, Jacob A., "Creole Urbanism : Searching for an Urban Future in the Flooded Streets of New Orleans », Space and Culture 9, 2006. 
Ces derniers ayant déjà fait l'objet d'une attention constante depuis 2005, cet article voudrait mettre au jour l'héritage du dualisme dans un autre discours : le discours festif. Plus précisément, il se donne pour objectif d'étudier la façon dont le carnaval met en scène les identités raciales à La Nouvelle-Orléans à partir de l'exemple des Mardi Gras Indians, tradition folklorique spécifique à la ville même si on trouve des échos dans d'autres lieux (Afrique de l'Ouest, Trinidad, Cuba, Haïti, Martinique, Panama, Honduras, Jamaïque et Brésil notamment) ${ }^{13}$.

Dans un premier temps, les processions organisées par ces tribus fictives $\mathrm{d}^{\prime}$ " Indiens noirs » seront envisagées au prisme des performance studies ${ }^{14}$, comme une porte dérobée vers un ailleurs racial, comme l'invention d'une identité et comme l'exercice du "droit à être un autre", pour reprendre une expression de Mikhail Bakhtine ${ }^{15}$. Ces mêmes défilés, ainsi que les six à neuf mois de préparatifs qui les précèdent, seront ensuite réinterprétés comme l'expression d'une identité noire conquérante et autonome qui se rit des règles instaurées par l'élite politique et économique blanche de La Nouvelle-Orléans dans le but de canaliser l'esprit festif et de marginaliser les pratiques culturelles ouvrières afro-américaines. Enfin, cet article reviendra sur la trêve conclue en 2011 entre la mairie de La Nouvelle-Orléans et les Mardi Gras Indians afin d'en démêler les implications en termes de relations de pouvoir depuis Katrina. L'hypothèse formulée en conclusion, selon laquelle la reconnaissance officielle accordée aujourd'hui aux pratiques festives des Indiens de Mardi Gras n'entraîne pas nécessairement leur « dépolitisation », sera testée sur le terrain en 2017-2018, dans le cadre d'une enquête ethnographique plus large sur le carnaval de La Nouvelle-Orléans.

13 Michael P. Smith dresse une liste non-exhaustive de ces correspondances transatlantiques dans Mardi Gras Indians, Gretna, Pelican Publishing, 2007 [1993], p. 107-108. Sur les blocs afroindiens de Bahia, lire Agier, Michel et Maria Rosário G. de Carvalho, « Nation, race, culture. Les mouvements noirs et indiens au Brésil », Cahier des Amériques latines n¹7, 1994, p. 107-124. Sur la tradition des mas amérindiens en Guadeloupe (mas-a-roukou du groupe carnavalesque Voukoum, par exemple) et à Trinidad, on consultera Mulot, Stéphanie, "La trace des masques : identité guadeloupéenne entre pratique et discours », Ethnologie française vol. 33 n¹, 2003, p. 111-122 ; Bellour, Hélène et Samuel Kinser, " Amerindian Masking in Trinidad's Carnival : The House of Black Elk in San Fernando », in TDR: The Drama Review vol. 42 n³ (numéro spécial "Trinidad and Tobago Carnival » coordonné par Milla Cozart Riggio), 1998, p. 147-169.

14 Inauguré par Richard Schechner et Victor Turner dans les années 1980, ce champ transdisciplinaire (voire post-disciplinaire, en ce qu'il s'emploie précisément à dépasser le découpage traditionnel du savoir en disciplines) envisage tout événement communautaire ritualisé (du barbecue dominical aux campagnes électorales) en termes de spectacle et de jeu théâtral. Cf. Schechner, Richard, Between Theater and Anthropology, Philadelphia, University of Pennsylvania Press, 1985. Préface de Victor Turner.

15 Voir Bakhtine, Mikhaïl, "Formes du temps et chronotope dans le roman », in Le principe dialogique, Paris, Seuil, 1981, p. 159. 


\section{L'invention d'une identité}

Les «Indiens noirs » de la Nouvelle-Orléans sont majoritairement des hommes ${ }^{16}$ issus de plusieurs quartiers ouvriers de la ville (Tremé, $7^{\text {th }}$ Ward, $9^{\text {th }}$ Ward, Carrollton) qui se regroupent en «tribus » et arborent, le jour de Mardi Gras, la nuit de la St-Joseph ${ }^{17}$ et trois autres dimanches de mars-avril («Super Sundays »), de splendides costumes chamarrés de plumes colorées, de perles et de sequins, cousus à la main au cours des six à neuf mois précédents. Leurs processions dans les ruelles ( backstreets ») de La Nouvelle-Orléans s'accompagnent de chants (moins d'une vingtaine au total, même si de nombreuses variantes existent) et de musique percussive à base de tambourins, clochettes et congas.

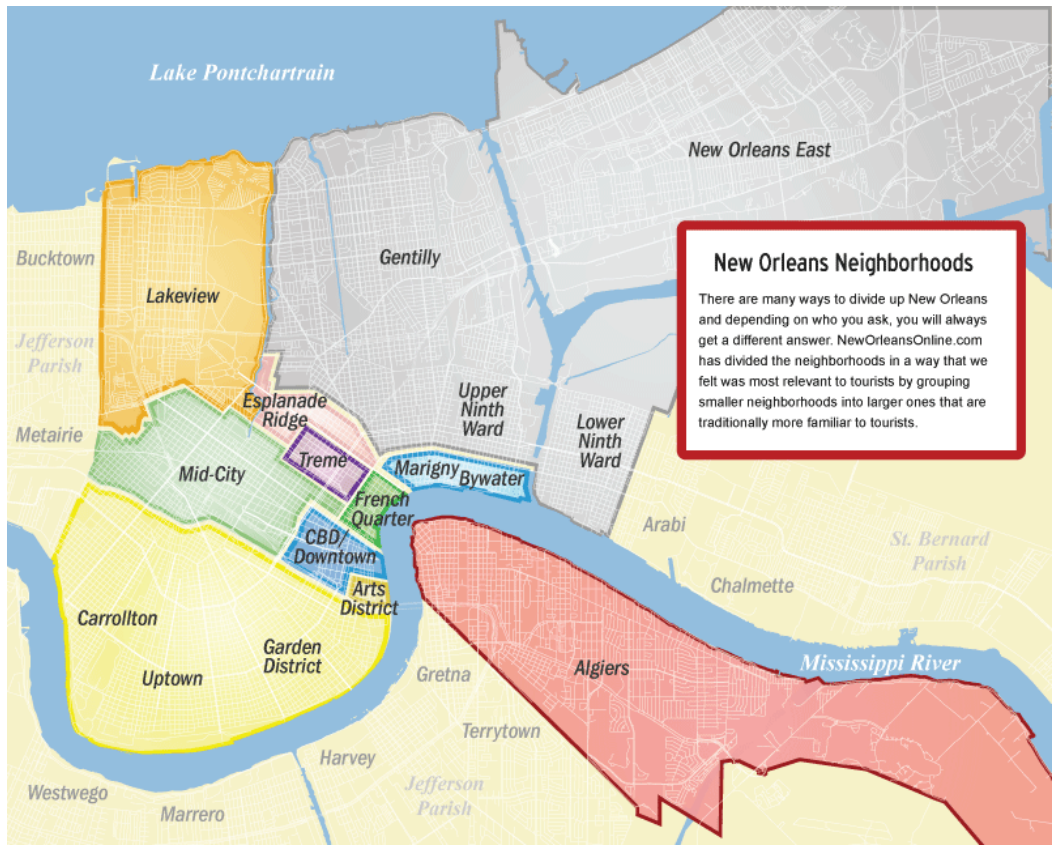

Carte des principaux quartiers de La Nouvelle-Orléans

telle que publiée sur le site officiel de la ville :

http://www.neworleansonline.com/tools/transportation/maps.html

16 En dépit de l'engagement militant de Cherice Harrison-Nelson, sœur de Donald Harrison, Jr. et «Big Queen » des Guardians of the Flame, en faveur de la féminisation des pratiques noires indiennes, les tribus ne comptent que peu de femmes parmi leurs membres. Lire à ce sujet Fensterstock, Alison, "Mardi Gras Indian Queens Stake Their Claim to an Essential Role in a Singular New Orleans Tradition », Times-Picayune, 2 mars 2014. Disponible en ligne : http://www.nola.com/ mardigras/index.ssf/2014/03/mardi_gras_indian_queens_stake.html. Consulté le 9 mars 2016.

17 Le choix de cette date est souvent attribué à un souci de discrétion. "La présence de nombreux Italo-Américains dans les rues pour la traditionnelle retraite aux flambeaux de la St-Joseph aurait permis aux Indiens de défiler sans attirer I'attention ». Disponible en ligne : http://www. mardigrasneworleans.com/supersunday.html. Consulté le 9 mars 2016. 
Quatre grandes théories ont été formulées afin d'expliquer ce phénomène de syncrétisme culturel sans qu'aucune ne prévale véritablement dans l'historiographie $^{18}$. Pour certains historiens et anthropologues, les rituels indiens noirs seraient le résultat d'interactions entre Noirs et Amérindiens entre 1800 et 1862 dans des lieux comme Congo Square où les esclaves d'ascendance ouest-africaine étaient autorisés à danser et jouer de la musique le dimanche ${ }^{19}$. Dans son livre Mardi Gras Indians, Michael P. Smith mentionne des récits de voyageurs publiés en 1823 et 1831 ainsi que des articles du New Orleans Times-Picayune qui attestent la présence d'Indiens à Congo Square au début du XIX siècle ${ }^{20}$. D’autres chercheurs comme Maurice Martinez ont, de leur côté, attribué l'existence des traditions indiennes du Mardi Gras aux mariages interraciaux contractés par leurs ancêtres. Le documentaire de 33 minutes que Martinez a produit et réalisé en 1976, The Black Indians of New Orleans, explique ainsi que les hommes et femmes qui défilent en costume indien le jour de Mardi-Gras sont en fait les descendants d'esclaves africains en fuite qui auraient trouvé refuge dans des tribus indiennes et se seraient mariés à certaines de leurs membres ${ }^{21}$. Sans rejeter $a$ priori cette hypothèse, pourtant fort peu documentée, Stephen Wehmeyer, Claude Jacobs et Andrew Kaslow ont préféré mettre en parallèle la revendication d'une identité «afro-indienne » avec la vénération des églises spiritualistes noires locales pour le chef amérindien Sauk Black Hawk, elle-même fondée sur l'enseignement de Leith Anderson, une femme mi-noire, mi-Mohawk qui prêchait la doctrine du "retour des esprits $»^{22}$. L'hypothèse la plus fréquemment avancée reste cependant celle de l'influence, sur la population noire de La Nouvelle-Orléans, des spectacles itinérants du Buffalo Bill's Wild West Show ${ }^{23}$. C'est en effet à la suite des représentations données par le Wild West de « Buffalo Bill » Cody à l'hiver 1884-1885

18 Berry, Jason, "Controversy Swirls Around Mardi Gras Indian Origins », New Orleans TimesPicayune, 17 février 1984.

19 Pour une histoire des pratiques artistiques à Congo Square, lire Evans, Freddi Williams, Congo Square : African Roots in New Orleans, Lafayette, University of Louisiana at Lafayette Press, 2011.

20 Smith, Michael P. Mardi Gras Indians, p. 81.

21 Un extrait du documentaire est visible en ligne à l'adresse : https://www.youtube.com/ watch?v=WYCeQ4r3DvM. Consulté le 9 mars 2016. La tribu des Guardians of the Flame, qui se décrit comme une "maroon society ", revendique haut et fort cet ancrage supposé dans la culture « marronne » louisianaise et caribéenne.

22 Wehmeyer, Stephen C., "Feathered Footsteps : Mythologizing and Ritualizing Black Indian Processions in New Orleans", in Carnival Art, Culture and Politics : Performing Life, Crichlow, Michaeline, dir., Londres, Routledge, 2013, p. 29-47 ; Jacobs, Claude et Andrew Kaslow, The Spiritual Churches of New Orleans : Origins, Beliefs, and Rituals of an African-American Religion, Knoxville, University of Tennessee Press, 1991 ; Welburn, Ron, "Mardi Gras Indians : Spiritualism or Indian Stereotype?», in American Indians and Popular Culture, vol. 1 : "Media, Sports, and Politics », DeLaney Hoffman, Elizabeth, dir., Santa Barbara, ABC-CLIO, 2012, p. 311-328.

23 Kinser, Samuel, Carnival American Style : Mardi Gras at New Orleans and Mobile, Chicago, University of Chicago Press, p. 97-105; Smith, Michael, « Buffalo Bill and the Mardi Gras Indians », Louisiana Cultural Vistas vol. 3 n³, automne 1992, p. 12-15. 
qu'est née la première tribu de Mardi Gras Indians, à savoir les «Creole Wild West ( (dont le nom renvoie par ailleurs explicitement au Hagenback Wallace Creole Wild West Show qui séjournait en Louisiane au même moment) ${ }^{24}$.

La question de savoir si les traditions afro-indiennes de La Nouvelle-Orléans sont le produit d'un métissage biologique ou non ne doit pas occulter un fait essentiel : l'« indianité » des Mardi Gras Indians est avant tout une construction. Rappelons tout d'abord, comme a pu le faire George Lipsitz en 1988, que les costumes et les coiffes exhibées par les différentes « tribus » sont modelés, non pas sur ceux des Séminoles, des Cherokees, des Choctaws, des Creeks ou des Chickasaw, originaires du Sud-Est des États-Unis, mais plutôt sur ceux des Indiens des Plaines (Sioux, Crows, Blackfoot, Arapaho, Kiowas, Cheyennes) exhibés et exaltés par la culture populaire $e^{25}$. De manière générale, les pratiques artistiques "black Indian » évoquent assez peu celles des Indiens de Louisiane, même si, dans un entretien accordé en 1958, Alice Zeno (mère du clarinettiste George Lewis) déclarait que les Mardi Gras Indians dansaient comme « de vrais Indiens » à la fin du XIX siècle (ce que Reid Mitchell a interprété comme une allusion aux traditions locales Choctaw) $)^{26}$, et même si Maurice Martinez a pu discerner dans la couronne de plumes portée par le «Big Chief » un rappel de celle arborée par les Indiens Natchez, installés le long des méandres du Mississippi ${ }^{27}$.

Le corpus musical de base des Indiens de Mardi-Gras est, de même, plus afro-américain qu'amérindien. Des chants comme "Handa Wanda ", "Get Out the Way » ou « Don't Like That Song » s'appuient sur une interaction entre Big Chief et chœur qui n'est pas sans rappeler le modèle responsorial (call and response), né en Afrique de l'Ouest et qui est devenu une des marques de fabrique de la musique liturgique afro-américaine ${ }^{28}$. Quant aux paroles d' 'Indian Red", le chant qui clôt toutes les répétitions des Mardi Gras Indians, elles ressemblent beaucoup plus au français ou à l'espagnol, langues pratiquées dans l'aire caraïbe, qu'à n'importe quelle langue amérindienne. Andrew Pearse a d'ailleurs retracé l'origine d'" Indian Red » dans un chant de carnaval trinidadien, "Indurubi », dont le titre lui-même proviendrait de l'espagnol indo rubi (Indien rouge) ${ }^{29}$. Jason Berry, Jonathan Foose et Tad Jones ont, de leur côté, interprété le titre du chant " Ma Day Cootie Fiyo », sur lequel s'ouvre la procession du Mardi Gras, comme voulant dire « tue celui qui se tient en travers de ta route ", sur la base d'une

24 Smith, Mardi Gras Indians, p. 105.

25 Voir Lipsitz, George, "Mardi Gras Indians : Carnival and Counter- Narrative in Black New Orleans », Cultural Critique n¹0 (automne 1988), p. 99-121.

26 Cité dans Mitchell, Reid, op. cit., p. 118.

27 Martinez, Maurice, op. cit.

28 Voir à ce sujet Martin, Denis-Constant, Le Gospel afro-américain. Des spirituals au rap religieux, Paris, Cité de la musique / Actes Sud, 2008 [1998].

29 Pearse, Andrew, "Carnival in Nineteenth Century Trinidad ", Caribbean Quarterly vol. 4 n³-4, 1956, p. 4-41. 
ressemblance entre « Ma » et le mot espagnol matar (tuer) ainsi qu’entre " Cootie Fayo » et qui tu es fijo (celui qui pour toi est immobile) ${ }^{30}$.

Au vu de ces libertés prises avec les codes culturels autochtones, il est difficile de ne pas faire le lien entre les rituels black Indian de La Nouvelle-Orléans et la pratique du "playing Indian » dont Deloria a retracé l'histoire dans un livre éponyme ${ }^{31}$. On y retrouve les mêmes généralisations erronées, les mêmes stéréotypes sur les peuples amérindiens que dans les westerns hollywoodiens ou certains manuels scolaires. Certaines nations indiennes refusent d'ailleurs pour cette raison de reconnaître aux Mardi Gras Indians toute légitimité artistique. Citons ici Ron Welburn, qui revendique lui-même une ascendance Cherokee/ Assateague et Gingaskin :

Pour certains Indiens d'Amérique du Nord, ni de rapides discussions ni l'historicisme universitaire ne pourront jamais améliorer, rectifier ou guérir l'offense suscitée par le spectacle de Noirs travestis en Indiens. Ces derniers éprouvent le même mélange de fascination et de révulsion à la vue du visage abondamment maquillé des « Mardi Gras Indians » que celui qu' ils éprouvent devant l'utilisation de figures indiennes comme mascottes par certaines équipes sportives. Ils ne comprennent pas comment des Noirs qui ont eux-mêmes fait l'expérience du racisme peuvent se laisser aller au jeu de la caricature ${ }^{32}$.

Le parallèle entre le «playing Indian » des Indiens de Mardi Gras et le «playing African » de l'association festive néo-orléanaise Zulu est tout aussi tentant. Dans les défilés carnavalesques du Zulu Social Aid and Pleasure Club, fondé par un groupe d'ouvriers noirs en 1916, le roi Zulu (reconnaissable à sa couronne de papier doré) et sa cour portent des perruques crépues et ébouriffées, des jupes de raphia ainsi qu'une épaisse couche de cire qui couvre l'ensemble de leur visage hormis la bouche et le contour des yeux. Dans les deux cas, on retrouve le même usage des stéréotypes et de l'hyperbole. L'usage du maquillage, commun à Zulu et aux Mardi Gras Indians, rappelle par ailleurs que les lois locales ont longtemps refusé aux organisations carnavalesques non reconnues par la municipalité (autrement dit, noires) le port du masque, y compris pendant le carnaval ${ }^{33}$.

30 Berry, Jason, Jonathan Foose et Tad Jones, Up From the Cradle of Jazz, Athens, University of Georgia Press, 1986, p. 218.

31 Deloria, Philip J., Playing Indian, New Haven, Yale University Press, 1998. À noter que Deloria ne mentionne les "Mardi Gras Indians » qu'une seule fois dans son livre, minorant ainsi leur importance dans I'histoire de cette pratique souvent considérée comme insultante.

32 Welburn, Ron, art. cit., p. 312-313. Dans un entretien avec Rebecca Solnit, Herreast Harrison, veuve de Donald Harrison Sr., a récemment rappelé l'accueil très froid réservé à son mari lors du grand rassemblement annuel des chefs indiens d'Amérique du Nord (Gathering of Nations) à Alburquerque en 1993. Cité dans Solnit, Rebecca et Rebecca Snedeker, dir., Unfathomable City: A New Orleans Atlas, Berkeley, California University Press, 2013, p. 105.

33 Roach, Joseph, Cities of the Dead. Circum-Atlantic Performance, New York, Columbia University Press, 1996, p. 270-271. 
Il ne faudrait pas pour autant exagérer la ressemblance entre ces deux pratiques. Tandis que l'usage du "blackface » par Zulu fonctionne comme un rappel outré et ironique de la «négritude » des Afro-Américains à la NouvelleOrléans, déjà parodiée dans les minstrel shows du XIX ${ }^{e}$ siècle, le "playing Indian " des travailleurs manuels noirs de La Nouvelle-Orléans se présente plutôt comme une échappée métaphorique vers un ailleurs racial ${ }^{34}$.

Nés dans les années 1880, alors que le " code noir » de Louisiane limitait déjà depuis plus de quinze ans la participation des Noirs à la vie économique locale ainsi que leur liberté de mouvement ou de rassemblement, les premiers groupes de Mardi Gras Indians se sont créés une identité indienne afin d'échapper aux contraintes juridiques de la ségrégation embryonnaire ${ }^{35}$. Encore aujourd'hui, les pratiques festives noires indiennes sont un défi au dualisme / binarisme racial qui fait des habitants de La Nouvelle-Orléans soit des Noirs soit des Blancs ${ }^{36}$. En perpétuant le souvenir des esclaves «marrons » qui auraient, selon la légende, trouvé refuge dans les tribus indiennes de la région ${ }^{37}$, les Black Indians dessinent les contours d'un « tiers espace » qui leur permet d'échapper au destin racial - autrement, au mélange d'essentialisation et de marginalisation dessiné par l'histoire locale. Au carrefour entre l'espace hégémonique et l'espace " hétérotopique " (le contre-site dont parlait notamment Michel Foucault ${ }^{38}$ ), leurs pratiques s'inscrivent dans un entre-deux, une " aire de liminalité paradoxale ${ }^{39}$ qui leur permet de faire entendre une parole " autre». L'usage de codes culturels amérindiens prend alors tout son sens en ce qu'il renvoie au concept de «frontière ", ce « tiers espace » où s'opère un mouvement constant

34 Sur la tradition du "blackface » et l'évolution de ses usages et de sa signification, lire Lhamon, Jr., William T., Raising Cain. Blackface Performance from Jim Crow to Hip-Hop, Cambridge, Harvard University Press, 1998. Traduit en français par Sophie Renaut sous le titre Raising Cain : représentations du blackface de Jim Crow à Michael Jackson, éditions Kargo et l'Éclat, 2004.

35 Parmi les études récentes sur la ségrégation progressive des espaces urbains (parcs municipaux, tramways, restaurants, hôpitaux, écoles, spectacles) à La Nouvelle-Orléans après la guerre de Sécession, citons entre autres Bennett, James B., Religion and the Rise of Jim Crow in New Orleans, Princeton, Princeton University Press, 2016 ; DeVore, Donald E., Defying Jim Crow : African American Community Development and the Struggle for Racial Equality in New Orleans, 1900-1960, Baton Rouge, Louisiana State University, 2015 ; Nystrom, Justin, New Orleans after the Civil War : Race, Politics, and a New Birth of Freedom, Baltimore, Johns Hopkins University Press, 2010 ; Simmons, LaKisha Michelle, Crescent City Girls : The Lives of Young Black Women in Segregated New Orleans, Chapel Hill, University of North Carolina Press, 2015.

36 Sur l'expression de ce modèle racial binaire nord-américain, voir Patterson, Orlando, "Four Modes of Ethno-Somatic Stratification : The Experience of Blacks in Europe and the Americas ", in Ethnicity, Social Mobility, and Public Policy : Comparing the USA and UK, Glenn C. Loury, Tariq Modood, Steven M. Teles, dir., Cambridge, Cambridge University Press, 2005, p. 67-122.

37 George Landry (1917-1980), "Big Chief » de la tribu des Wild Tchoupitoulas, expliquait ainsi à ses neveux, les frères Neville, que « les tribus noires indiennes avaient été créées afin de rendre hommage à l'aide apportée par les nations indiennes aux Noirs pendant la période de l'esclavage ». Cité dans Welburn, Ron, art. cit., p. 320.

38 Foucault, Michel, "Des espaces autres ", in Dits et écrits, tome IV, Daniel Defert et François Ewald, dir., Paris, Gallimard, 1994, p. 752-762.

39 Westphal, Bertrand, La géocritique : réel, fiction, espace, Paris, Minuit, 2007, p. 116. 
de déterritorialisation / reterritorialisation permettant l'invention de traditions et de « communautés imaginées ${ }^{40}$.

\title{
Une tradition affirmative
}

Le choix de l'échappée vers un ailleurs racial, combiné au secret qui entoure les pratiques festives des black Indians (longtemps confinées aux "backstreets ", celles-ci n’ont rejoint « Main Street » qu’à partir des années 1960, lorsque la ville a commencé à promouvoir son identité caribéenne pour attirer de nouveaux touristes ${ }^{41}$ ) peut à certains égards s'apparenter à un évitement de la confrontation. Mais on peut également l'envisager comme une forme de contestation, ainsi que l'ont fait R.G. Kelley dans Race Rebels et George Lipsitz dans Time Passages ${ }^{42}$.

En se déguisant en autochtones stylisés, les ouvriers et artisans noirs de La Nouvelle-Orléans s'appuient sur le symbolisme associé à l'Indien des Plaines. Vaincu par l'homme blanc à la fin du XIV siècle, celui-ci a pour autant survécu dans l'imaginaire collectif américain comme une figure de résistance au colonialisme, de prouesse guerrière et de virilité. Les chants des tribus afro-indiennes de La Nouvelle-Orléans, et notamment «Indian Red », perpétuent cette fierté masculine :

\author{
Chour: We are the Indians, Indians, \\ We're the Indians of the Nation. \\ The wild, wild creation. \\ Soliste: We won't bow down \\ Chour: We won't bow down, \\ Soliste: Down on the ground \\ Chour: That dirty ground \\ Tous: Oh how I love to hear them call my Indian Red!
}

40 Anderson, Benedict, Imagined Communities : Reflections on the Origin and Spread of Nationalism, New York, Verso, 2006 [1983] ; Hobsbawm, Eric et Terence O. Ranger, The Invention of Tradition, Cambridge, Cambridge University Press, 1992. Sur l'image de la "frontière » dans la culture américaine depuis Frederick Jackson Turner, on se reportera à White, Richard et Patricia Nelson Limerick, The Frontier in American Culture, Berkeley, University of California Press, 1994.

41 Sur ce point, voir Becker, Cynthia, « New Orleans Mardi Gras Indians. Mediating Racial Politics from the Backstreets to Main Street ", African Arts vol. 46 n², 2013, p. 42-43.

42 Dans Race Rebels : Culture Politics and the Black Working Class, I'historien Robin D. G. Kelley parle de «formes cachées de résistance » à propos de certaines pratiques culturelles noires nées dans le Sud de I'après-Reconstruction. Dans Time Passages : Collective Memory and American Popular Culture, I'historien et sociologue George Lipsitz a, de son côté, mis en lumière la façon dont les identités politiques pouvaient s'inscrire dans l'expression culturelle des groupes ethnoraciaux minoritaires. 
Au-delà du symbolisme amérindien, la constitution d'une tradition artistique et matérielle spécifique à la classe ouvrière noire néo-orléanaise est vecteur d'affirmation identitaire. Se déguiser en Indien est source de prestige pour les résidents des quartiers ouvriers noirs de La Nouvelle-Orléans. Leur petit nombre, 300 à 400 personnes, et leur implication dans la vie locale fait d'eux des figures respectées dans le voisinage immédiat. La valorisation du statut d'Indien se fait sans doute au détriment de leur ascension socio-économique (le coût de fabrication d'un costume s'élevant souvent à plusieurs milliers de dollars), mais elle leur apporte, en contrepartie, la reconnaissance de leur valeur en tant qu'individus braves, vaillants et indomptables.

L'exaltation de la figure du Grand Chef que l'on trouve dans certains chants comme " My Big Chief Got a Golden Crown » ou " Big Chief » peut laisser penser que les Mardi Gras Indians glorifient une vision autocratique du pouvoir. Pourtant, derrière tous les chants célébrant la puissance et la fierté du Big Chief, il faut comprendre que c'est la tribu entière qui refuse de s'abaisser et de courber l'échine. Le groupe s'identifie à la grandeur de son meneur, qui en est l'emblème charismatique. Autrement dit, l'organisation des tribus noires indiennes ne renvoie pas à une structure pyramidale de type occidental, mais plutôt à un agencement de type tribal, à la fois proche de celui des Amérindiens et de celui de certaines sociétés africaines ${ }^{43}$.

Lautonomie de la culture afro-indienne de La Nouvelle-Orléans se traduit par la quasi-absence de références aux sources européennes du carnaval, aux groupes carnavalesques les plus connus ou aux quartiers blancs de la ville dans le répertoire black Indian ${ }^{44}$. La culture festive de ces derniers se construit indépendamment de celle de l'élite blanche de la ville et se concentre sur les quartiers et les rues dont elle est issue, ainsi qu'en témoigne le nom des tribus suivantes : " $7^{\text {th }}$ Ward Hunters ", " $9^{\text {th }}$ Ward Hunters ", "Wild Treme », "Carrollton Hunters ", «Wild Magnolias », «Wild Tchoupitoulas ».

Ce repli sur l'échelon du voisinage n'exclut pas les interactions à l'échelle de la ville tout entière. Centrale dans la géographie mentale des Indiens noirs est en

43 Chacune des trente-huit tribus noires-indiennes actuelles inclut ainsi un "Spy Boy » (éclaireur), un "Flag Boy » (porte-étendard) et un « wildman » (homme sauvage, héritier des bêtes à cornes des mascarades africaines), dont les rôles respectifs (alerter verbalement le « Big Chief » de l'arrivée d'une tribu rivale, communiquer gestuellement avec lui, effrayer et menacer les ennemis potentiels) sont à la fois différents et complémentaires. Entre eux circule la " second line ", faite de dizaines de participants non costumés qui accompagnent la procession de leurs danses, chants et musique.

44 Tout juste peut-on trouver une référence à Rex, organisation carnavalesque blanche créée en 1872, dans une version de "Golden Crown » enregistrée en 1972 : "Well Rex is the Ruler, Mardi Gras Day / My big chief got the golden crown / I'm an Injun ruler, make Kil-a-way / Big Chief's got the golden crown / Kil-a-way / pok-y-way : get out of the way! Not Rex but this black Indian is the true carnival ruler, a man of executive / executionary power ». Citée dans Kinser, Samuel, "Violence Ritually Enjoined: The Mardi Gras Indians of New Orleans », Cahiers de littérature orale $\mathrm{n}^{\circ} 37,1995$, p. 133. 
effet la rivalité entre tribus nées au nord de Canal Street ou, aujourd'hui, de la voie express Pontchartrain (Yellow Jackets, White Eagles, Yellow Pocahontas, Cherokee Hunters, Golden Stars) et tribus nées au sud (Black Eagles, Golden Eagles, Apache Hunters, Wild Magnolias, Golden Arrows, Iroquois Hunters, Cheyenne Hunters). Cette rivalité entre "downtown » et "uptown", dont les origines sont mal connues (Michael Smith pense qu'elle s'explique par la revendication ou non d'une ascendance afro-créole ${ }^{45}$ ), s'est traduite historiquement par de nombreux règlements de compte entre tribus.

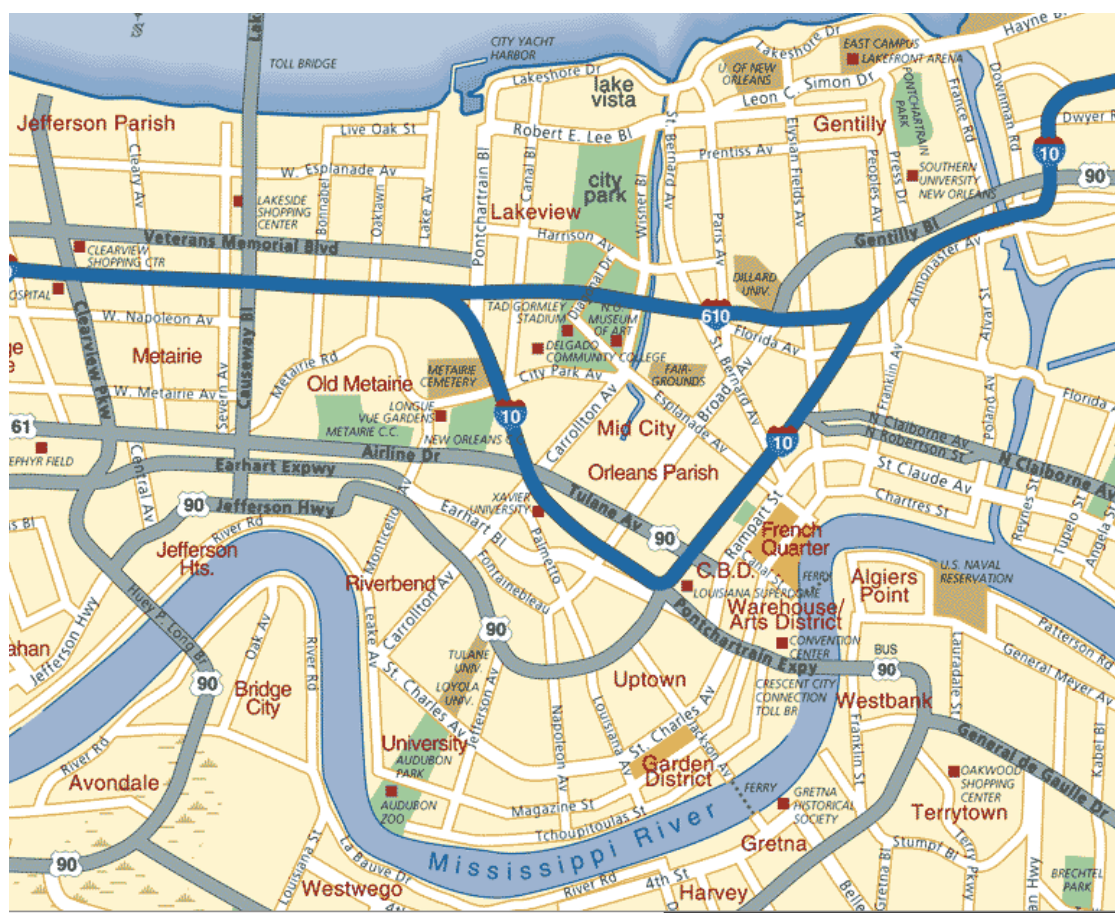

Carte de la Nouvelle-Orléans

où l'on distingue Canal Street, la Pontchartrain Express Way et la séparation que ces artères opèrent entre "Uptown » et " Downtown ». Source : http://www.mappery.com/map-of/New-Orleans-Neighborhood-Map

Des années 1880 aux années 1940, les processions des Indiens de Mardi Gras étaient souvent prétexte à des affrontements entre bandes rivales (alors appelées "gangs »). Armés de machettes, de rasoirs, et parfois d'armes à feu dissimulées sous leurs costumes, les Indiens se défiaient, parfois sur un champ de bataille prévu à cet effet (un terrain vague situé à l'intersection des rues Claiborne et

45 Smith, Michael P., op. cit., p. 122. Voir également les explications très éclairantes de Simmons, LaKisha Michelle, op. cit., p. 32-33. 
Poydras), parfois lors des défilés eux-mêmes. Pour l'ethnomusicologue David Elliot Draper, ce recours au duel de champions avait pour but de régler les conflits internes à la communauté noire : " Par le passé, lorsque les tribus s'adonnaient encore au combat physique, leurs membres semblent avoir joué le rôle institutionnel de "champions", et cette pratique représentait un moyen de résoudre les rivalités à l'échelle du voisinage ou du quartier ${ }^{46}$. Sans surprise, un tel comportement suscitait la peur chez les personnes étrangères à la tradition, et la police municipale se gardait bien d'intervenir. " Je vous parle d'hommes qui vous auraient tué avec leur poing. Des tueurs froids... À l'époque [les années 1920], les gens fuyaient devant les Indiens », rappelait un chef indien noir dans les années $1980^{47}$.

Depuis les années 1940, en lien avec l'avènement du tourisme de masse à La Nouvelle-Orléans ${ }^{48}$ et sous l'influence du chef Allison « Tootie » Montana, petit-neveu de Becate Batiste, fondateur de la tribu Creole Wild West, le combat physique s'est vu progressivement remplacé par une rivalité de nature esthétique, ainsi qu'en atteste cet extrait du chant " Meet De Boys on de Battlefront », créé par le Chef Jolley (George Landry) de la tribu Wild Tchoupitoulas au milieu des années 1970 :

\section{Well the prettiest little thing that I ever see Is the Mardi Gras Indian down in New Orleans \\ Well he sewed all night and he sewed all day \\ On Mardi Gras morning went all the way.}

Lobjet de l'attention ici n'est pas la force ou le courage des combattants indiens, mais la splendeur de leurs costumes. Autrement dit, on assiste à une inversion des codes sexuels en vigueur dans le reste de la société américaine : les hommes revêtus de leurs atours guerriers sont "pretty" et non pas "fierce " ${ }^{49}$.

En lien avec ce transfert ou, pour utiliser un terme freudien, cette « sublimation» (que l'on définira ici comme un mécanisme de transformation de certains instincts vers des objectifs considérés comme plus légitimes socialement), les valeurs de solidarité et de communauté sont devenues prépondérantes dans les traditions noires indiennes. Dans les années 1970, les Black Indians ont ainsi

46 Draper, David Elliot, "The Mardi Gras Indians : The Ethnomusicology of Black Associations in New Orleans ", thèse de doctorat, Tulane University, 1973, p. 37.

47 Cité dans Berry, Jason, Jonathan Foose et Tad Jones, op. cit., p. 212.

48 Sur ce point, on consultera Gotham, Kevin Fox, Authentic New Orleans : Tourism, Culture and Race in the Big Easy, New York, New York University Press, 2007 ; Gotham, Kevin Fox, "Marketing Mardi Gras : Commodification, Spectacle, and the Political Economy of Tourism in New Orleans ", Urban Studies vol. 39 n¹0, 2002, p. 1735-1756 ; Souther, Mark, New Orleans on Parade : Tourism and the Transformation of the Crescent City, Baton Rouge, Louisiana State University Press, 2006.

49 Cette insistance sur le caractère « joli » des costumes pourrait également être lue comme un rite de cumul plus que d'inversion, autrement dit une mise en scène de la complémentarité des sexes. Il s'agit là d'une hypothèse qu'il faudrait sans doute explorer plus avant. 
créé une association afin de promouvoir et transmettre leurs pratiques festives et artistiques : la Mardi Gras Indian Association ${ }^{50}$. Si, pour les adolescents, celle-ci fait avant tout office de club de loisirs, pour les adultes, c'est un groupe d'entraide qui peut servir d'assurance en cas d'enterrement d'un membre nécessiteux. Cette notion de solidarité à l'échelle du groupe se double d'une solidarité individuelle entre certains membres, qu'illustre bien l'expression "my boy", née dans les quartiers pauvres de La Nouvelle-Orléans au début du $\mathrm{xx}^{\mathrm{e}}$ siècle. Plus forte que l'appellation «meilleur ami », elle suppose chez les Noirs y souscrivant des obligations de solidarité rapprochée. Dans le cas d'un emprisonnement par exemple, l'« ami » va avoir comme préoccupation le bien-être de l'autre et de sa famille. Cette notion d'aide mutuelle entre amis peut devenir vitale dans des quartiers au fort taux de délinquance. Samuel Kinser la relie aux institutions mutualistes mises en place à partir du XVIII ${ }^{\mathrm{e}}$ siècle par les Yoruba en Afrique de l'Ouest (ajo, esusu $)^{51}$ puis transposées à La Nouvelle-Orléans sous la forme de sociétés funéraires (burial societies) et de sociétés de secours mutuel (social aid and pleasure clubs) $)^{52}$.

La sublimation dont on vient de parler a, bien sûr, ses limites. Les règlements de compte entre tribus noires-indiennes n'ont pas entièrement disparu. À titre d'exemple, le fils du chef de la tribu «Black Feather », Lionel Delpit Jr., ainsi que sa compagne ont été tués en décembre 2015 au cours d'une fusillade sans qu'aucun suspect ne soit appréhendé ${ }^{53}$. Il n'en demeure pas moins qu'il faut remonter à 1981 pour retrouver la trace d'une agression physique d'un Mardi Gras Indian sur un autre lors d'une procession festive ${ }^{54}$. Tout se passe comme si, de l'expression violente de tensions intra-communautaires, les rituels black Indian étaient devenus les vecteurs d'une affirmation identitaire.

De fait, en défilant dans les rues de La Nouvelle-Orléans sans autorisation et en refusant toute protection policière, les Indiens de Mardi Gras combinent souci du secret et de la transmission, repli géographique et ouverture à l'autre, agressivité et entraide. Ils donnent une forme visible, spectaculaire, à une tradition faite de travail collectif minutieux à domicile et de répétitions dominicales dans les bars du quartier (appelées "practices » en écho aux contraintes qui pèsent sur les pratiques culturelles noires). Le carnaval, traditionnellement décrit comme

50 Rebaptisée Mardi Gras Indian Council en 1987, c'est elle qui organise, entre autres, le défilé du dimanche qui précède ou suit la Saint-Joseph ("Super Sunday »).

51 Kinser, Samuel, Carnival American Style, p. 114.

52 Sur ces sociétés, lire Abrahams, Roger D., Nick Spitzer, John F. Szwed et Robert Farris Thompson, Blues for New Orleans: Mardi Gras and America's Creole Soul, Philadelphie, University of Pennsylvania Press, p. 70.

53 Cf. http://www.nola.com/crime/index.ssf/2015/12/he_would_have_been_one_of_the.html. Consulté le 9 mars 2016.

54 Sur cette agression, lire Bragg, Rick, " Another Battle of New Orleans : Mardi Gras ", New York Times, 19 février 1995. Disponible en ligne : http://www.nytimes.com/1995/02/19/us/anotherbattle-of-new-orleans-mardi-gras.html?pagewanted=all. Consulté le 9 mars 2016. 
un espace-temps liminaire où se vit un dédoublement de soi, une rupture avec le monde des dominations ordinaires au travers de la parodie bouffonne, de la défiguration parodique ou de la dramatisation excessive, devient ici un espacetemps de subjectivation au sens où des sujets, à un moment donné et dans un lieu propice à cette expression, émergent en imposant leur parole contre l'identité inscrite dans le langage qui les soumet, les infériorise ou les diminue.

Au total, le Black Indian apparaît comme une figure doublement équivoque et métaphorique. Comme le rappelle Karen Williams, il exprime sa propre culture en se déguisant en Amérindien et, de ce fait, mystifie les Blancs qui n'y voient qu'un spectacle, alors qu'il s'agit d'une forme d'affirmation identitaire métaphorique de ses origines africaines : "L'homme noir ne se contente pas de se comparer à l'Indien lorsqu'il endosse le costume des 'premiers" Américains : il se déguise en Indien afin de devenir ce qu'il est (ou a été), à savoir un Africain ${ }^{55}$. Les rituels afro-indiens seraient ainsi cette manière détournée pour les Noirs de continuer à vivre leurs traditions sans provoquer la répression de la part des Blancs. « Plusieurs centaines d'années de coexistence dans le Nouveau Monde ont fait émerger un accord tacite entre Noirs et Blancs : en échange de la suppression ou de la dissimulation de leurs racines rituelles, religieuses ou politiques, un système festif afro-américain relativement autonome a pu émerger », explique Kinser ${ }^{56}$.

On rejoint ici le concept d' "infra-politique des subalternes » forgé par James C. Scott dans La domination ou les arts de la résistance à partir d'enquêtes sur les rapports de classe dans la société paysanne en Malaisie ${ }^{57}$. De Weber à Bourdieu, en passant par Gramsci, l'ordre social est le plus souvent pensé comme le fruit d'une croyance : les dominés acceptent leur situation subalterne car ils croient à la légitimité du pouvoir et aux idéologies dominantes qui présentent l'ordre social pourtant inégalitaire comme naturel et juste. Or, pour Scott, une telle conception recèle des « erreurs fondamentales ». Il faut, notamment, refuser les théories de la «fausse conscience » qui postulent que la domination idéologique des élites est si efficace que leurs valeurs et leurs représentations sont nécessairement adoptées et incorporées par les dominés. À ses yeux, dans toutes les sociétés, les dominés disposent de ressources symboliques pour penser des ordres alternatifs, qu'il s'agisse d'imaginer une inversion de l'ordre social ou une société sans inégalités. Il faut ainsi chercher à appréhender ce qu'il appelle le « texte caché » des groupes

55 Williams, Karen L., Images of Uneasy Hybrids : Carnival and New Orleans, thèse, Emory University, 1992, p. 87.

56 Kinser, Samuel, op. cit., p. 57.

57 Scott, James C., op. cit.. Voir également, du même auteur, Weapons of the Weak : Everyday Forms of Peasant Resistance, New Haven, Yale University Press, 1985, Decoding Subaltern Politics. Ideology, Disguise, and Resistance in Agrarian Politics, London, Routledge, 2012, ainsi que "Infrapolitics and Mobilizations : A Response by James C. Scott », Revue française d'études américaines $\mathrm{n}^{\circ} 131,2012$, p. 112-117. 
dominés à travers lequel ceux-ci tentent d'exprimer griefs et défiance à l'égard du pouvoir. Ce faisant, il propose de placer au centre de l'analyse des formes de protestation des comportements habituellement invisibles et délaissés par l'histoire et la sociologie : cultures populaires, pratiques religieuses dissidentes, ragots, rumeurs, formes à peine perceptible de désobéissances comme l'humour, l'ironie ou les provocations vestimentaires. L'exemple des rites festifs des Mardi Gras Indians illustre parfaitement cette idée de contestation des hiérarchies de pouvoir par la création d'une « contre-tradition » dans laquelle les puissants sont raillés et l'ordre social critiqué, sans que ces formes de résistance ne prennent une tournure explicitement politique.

\section{Une pacification à double tranchant}

Jusque dans les années 1960, les tribus d'Indiens de Mardi Gras sont parvenues à échapper au regard inquisiteur du pouvoir en défilant en secret dans les ruelles des quartiers noirs de La Nouvelle-Orléans plutôt que sur les grandes artères empruntées par l'establishment carnavalesque. À l'écart du centre-ville et de l'activité touristique (à l'époque centrée sur Bourbon Street), les traditions afro-indiennes se sont construites en opposition à la conception fondamentalement matérialiste et élitiste de la fête promue par des krewes ${ }^{58}$ comme Comus, Proteus, Momus, Rex, Bacchus, Endymion ${ }^{59}$, confectionnant leurs costumes sans machine à coudre et invitant la foule à participer au défilé en formant une " second line " (supra, note 43).

L'élection à la tête de la ville de Maurice Edwin Landrieu (père de l'ex-sénatrice de Louisiane Mary Landrieu et de l'actuel maire de la ville, Mitch Landrieu) a quelque peu changé la donne. Parallèlement à son engagement en faveur de la promotion sociale et politique des Afro-Américains (il a fait retirer le drapeau confédéré des bâtiments municipaux, nommé des Noirs dans son équipe, y compris le futur maire de la ville Sidney Barthelemy, et fait passer le

58 Krewe est le nom que se donnent les principales associations carnavalesques qui défilent dans les rues de La Nouvelle-Orléans depuis 1857. Historiquement dominées par l'élite blanche et masculine de la ville, elles ont vu leur composition se diversifier considérablement à partir des années 1960. Sur leur histoire (dont de larges pans demeurent méconnus), lire Gill, James, Lords of Misrule: Mardi Gras and the Politics of Race in New Orleans, Jackson, University Press of Mississippi, 1997 ; Kinser, Samuel, op. cit. ; Mitchell, Reid, All On a Mardi Gras Day. Episodes in the History of New Orleans Carnival, Cambridge, Harvard University Press, 1999 ; O'Neill, Rosary, New Orleans Carnival Krewes : The History, Spirit and Secrets of Mardi Gras, Charleston, The History Press, 2014. Voir également le documentaire de Rebecca Snedeker et T. Watson, By Invitation Only, Palmetto Pictures et New Day Films, 2006.

59 Les quatre premiers sont nés dans les années 1850-1880, incarnent l'aristocratie blanche du carnaval et cultivent encore aujourd'hui le secret ; les deux derniers sont apparus dans les années 1960 et ont fait de la surenchère leur marque de fabrique, déversant des milliers de colliers, doublons et bibelots à leur effigie sur les foules (d'où leur surnom de «méga-défilés ", traduction de « super-krewes»). 
pourcentage de Noirs employés par la ville de 19,4\% en 1970 à $43 \%$ en 1978), «Moon » Landrieu était en effet déterminé à faire oublier la réputation sulfureuse de La Nouvelle-Orléans et à attirer une clientèle touristique plus familiale ${ }^{60}$. Par ricochet, la police est devenue beaucoup moins tolérante à l'égard des infractions commises par les Mardi Gras Indians (parmi lesquelles figure, rappelons-le, le refus de solliciter une quelconque autorisation pour défiler dans les rues). Une chanson comme " Jock-A-Mo », rappelle à juste titre qu'il n'était pas rare dans les années 1970 qu'un chef indien passe quelques jours en prison la nuit de Mardi Gras ou de la St-Joseph.

\section{Boys, I was walking on Canal Street \\ Policeman blowed his whistle \\ Up jumped Baby on a Mardi Gras Day \\ With a great big shining pistol ${ }^{61}$.}

Les tensions entre police et Indiens ont connu leur apogée le 19 mars 2005, lorsque le New Orleans Police District a décidé de mettre un terme à un rassemblement d'une centaine d'entre eux dans le parc A. L. Davis, situé en face de l'ancienne cité HLM Magnolia. Ainsi que l'a relaté Katy Reckdahl dans un article du magazine local Gambit, les participants se sont vus donner le choix de quitter leur costume ou bien de se faire arrêter ${ }^{62}$. Les black Indians ont dénoncé cette alternative inique en juin 2005 lors d'une audition devant le Conseil municipal. La séance a malheureusement tourné cours lorsque le Grand Chef Allison « Tootie » Montana s'est écroulé en plein milieu de son intervention, victime d'une crise cardiaque à l'âge de 84 ans ${ }^{63}$.

Le passage de l'ouragan Katrina deux mois plus tard n'a pas apaisé les relations entre Mardi Gras Indians et forces de l'ordre, bien au contraire. Les scènes d'intimidation policière se sont multipliées et plusieurs rencontres rituelles entre Big Chiefs se sont vues dispersées après six heures du soir en 2009, 2010 et $2011^{64}$. Cette année-là, cependant, une commission municipale ad hoc sur les

60 Sur ce point, voir Souther, Mark, New Orleans on Parade, p. 182.

61 Citée dans Kinser, art. cit., p. 134. Dans la série télévisée Tremé (saison 1 épisode 8), le Grand Chef des Guardians of the Flame, Albert Lambreaux, passe, lui aussi, la nuit de Mardi Gras en prison pour avoir occupé illégalement un appartement du complexe HLM Calliope (Calliope projects).

62 Reckdahl, Katy, "St. Joseph's Night Gone Blue », Gambit, 29 mars 2005. Disponible en ligne : http://www.bestofneworleans.com/gambit/st-josephs-night-gone-blue/Content?oid=1244049. Consulté le 9 mars 2016.

63 Sur cet épisode tragique de l'histoire récente des "Mardi Gras Indians », voir le documentaire de Lisa Katzman, Tootie's Last Suit, New York, Pomegranate Productions, 2006.

64 Le 9 mars 2010, par exemple, une voiture de fonction a maintenu sa sirène allumée jusqu'à la fin de la rencontre rituelle entre deux Grands Chefs, Victor Harris de la tribu Spirit of FiYiYi et David Montana des Yellow Pocahontas. Cf. Reckdahl, Katy, « New Orleans Police Handling of Mardi Gras Indian Events Draws Praise/Criticism », Times-Picayune, 20 mars 2010. Disponible en ligne : http://www.nola.com/crime/index.ssf/2010/03/new_orleans_police_handling_of.html. 
nuisances sonores (Sound Ordinance Task Force) dirigée par Kristin Gisleson Palmer a proposé une révision de l'édit limitant le niveau sonore des rues de La Nouvelle-Orléans dans un sens "plus gérable par la police et plus respectueux des musiciens ", selon les termes d'un employé de la mairie ${ }^{65}$. Quelques mois plus tard, lors d'une réunion de la commission municipale chargée des questions de gouvernance (City Council's Governmental Affairs Committee) présidée par Susan Guidry, forces de l'ordre et Indiens ont enfin confronté leurs points de $v e^{66}$. Le commissaire adjoint Kirk Bouyelas, devant l'ensemble des lieutenants, a finalement promis que le NOPD cesserait d'interpeller les Mardi Gras Indians en l'absence de délit caractérisé et abandonnerait l'usage des sirènes ou des gyrophares pour les forcer à quitter les rues. Bouyelas a par ailleurs ordonné à ses subordonnés de patrouiller les rues à pied plutôt qu'en voiture de fonction lors des processions afro-indiennes. Enfin, il a suggéré que des formations à destination des officiers soient organisées dans les écoles de police afin d'inculquer aux jeunes recrues une « attitude positive » vis-à-vis des traditions noires indiennes. Lorsque la police a en revanche suggéré que les tribus indiennes fassent une demande de permis via le site de la municipalité pour avoir le droit de défiler dans les rues, Jerome Smith, fondateur de l'association Tambourine and Fan, destinée à faire découvrir aux jeunes de La Nouvelle-Orléans les traditions culturelles noires de la ville, a répliqué : "On ne peut pas contrôler un oiseau. Les rues appartiennent au peuple » ("You cannot police a bird. The streets belong to the people »). Le point a alors été supprimé de l'ordre du jour des négociations.

Consulté le 9 mars 2016. Le jour de Mardi-Gras 2011, les forces de l'ordre ont interrompu une rencontre entre les membres des Red Hawk Hunters et des $9^{\text {th }}$ Ward Hunters. Watts, Lewis et Eric Porter, New Orleans Suite : Music and Culture in Transition, Berkeley, University of California Press, 2013, p. 52.

65 Blumenfeld, Larry, "Beyond Jazzfest, Ruffled Feathers in New Orleans », Village Voice, 22 juin 2011. Disponible en ligne : http://www.villagevoice.com/2011-06-22/music/beyondjazzfest-ruffled-feathers/2/. Consulté le 9 mars 2016. Pour une analyse des débats récents sur les nuisances sonores et la musique de rue dans le centre-ville historique de La Nouvelle-Orléans, voir Le Menestrel, Sara, "Live Music and Noise Debates in New Orleans Post-Katrina ", Oxford Handbooks Online, novembre 2014. Disponible en ligne : http://www.oxfordhandbooks.com/ view/10.1093/oxfordhb/9780199935321.001.0001/oxfordhb-9780199935321-e-115?print=pdf. Consulté le 9 mars 2016.

66 Maldonado, Charles, "The Mardi Gras Indians and the NOPD », Gambit, 14 février 2012. Disponible en ligne : http://www.bestofneworleans.com/gambit/the-mardi-gras-indians-andthe-nopd/Content?oid=1956440. Consulté le 9 mars 2016. 


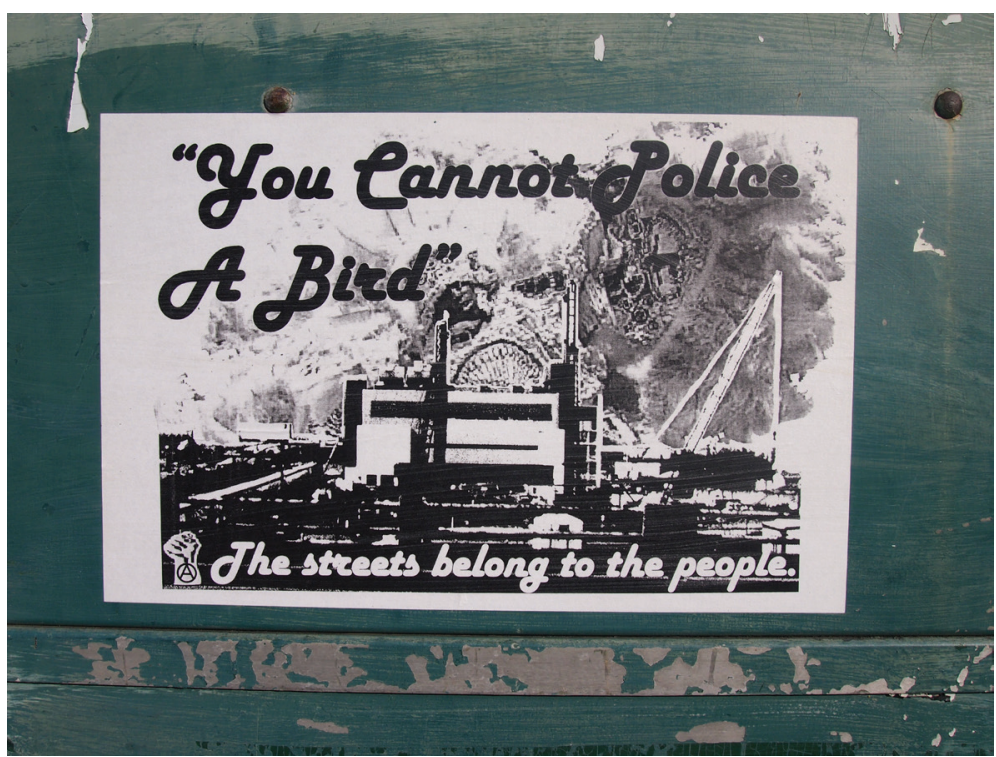

Affiche arborant les propos de Jerome Smith, placardée dans le centre-ville peu avant la nuit de la St-Joseph 2012

Contrairement aux craintes d'un grand nombre de chefs indiens, les promesses faites par le NOPD ont été tenues et la nuit de la St-Joseph 2012 n'a été la scène d'aucune échauffourée entre la police et les Mardi Gras Indians ${ }^{67}$. Ce calme n'a, d'ailleurs, pas été troublé depuis maintenant quatre ans. «Les policiers nous aident plus qu'ils ne nous gênent ", affirmait récemment le Big Chief des Wild Magnolias, Gerard «Bo » Dollis ${ }^{68}$. On peut, bien sûr, se réjouir de cette pacification, en ce qu'elle va de pair avec la reconnaissance de l'importance des traditions noires indiennes dans le patrimoine culturel de la ville. Au Japon, l'on dirait que les Mardi Gras Indians se sont hissés au rang de « trésor national vivant ». Certains membres de la communauté Black Indians tirent néanmoins la sonnette d'alarme : l'apaisement des relations interraciales à La Nouvelle-Orléans ne conduit-il pas nécessairement à une «muséification » des pratiques festives noires?

Depuis les années 1970, les enregistrements de chants indiens (en studio ou dans les rues) sont devenus des best-sellers, les costumes cousus patiemment à la main pendant un an se sont vus exposés dans beaucoup de musées américains

67 Reckdahl, Katy, "Crackdown a Decade Ago Touched Off Powder Keg ", The New Orleans Advocate, 19 mars 2015. Disponible en ligne : http://www.theneworleansadvocate.com/ news/11880449-123/decade-after-st-josephs-day. Consulté le 9 mars 2016.

68 Cité dans Reckdahl, Katy, «Decade After St. Joseph's Day Incident, Relations Between Police and Mardi Gras Indians Much Improved », New Orleans Advocate, 18 mars 2015. Disponible en ligne : http://www.theneworleansadvocate.com/news/11880449-123/decade-after-st-josephsday. Consulté le 9 mars 2016. 
(de la Smithsonian Institution en 1985 à la House of Dance and Feathers depuis 2003 en passant par le Backstreet Museum depuis 1999) alors que la tradition était jusque-là de les brûler au soir de la St. Joseph, et certains groupes de Black Indians ont été invités à se produire lors de conférences ou de festivals folkloriques (le New Orleans Jazz \& Heritage Festival, notamment). Au cours de l'hiver qui a suivi le passage de Katrina, les Mardi Gras Indians ont été érigés en symbole de résilience, et c'est à ce titre que dix-neuf d'entre eux ont participé à la tournée « Higher Ground » organisée par le ministère américain des Affaires étrangères pour remercier les pays qui avaient offert leur aide aux États-Unis (Japon, Sri Lanka, Inde, Émirats d'Arabe Unie, Qatar, Maroc, Sénégal, Roumanie ${ }^{69}$.

Ce déplacement de la marge vers le centre s'est accompagné d’une autonomisation progressive des traditions Mardi Gras Indians à l'égard de leur contexte social de naissance. En s'exhibant en dehors du cadre du carnaval (dans les musées, dans les festivals, à la télévision, à l'étranger), celles-ci ont acquis une dimension artistique qui dépasse leur contexte de production. Donald Harrison, Sr. des Guardians of the Flame (1933-1998), Allison Montana des Yellow Pocahontas (1922-2005), Larry Bannock des Golden Star Hunters (1948-2014) et Bo Dollis ont, par exemple, souvent été mis en avant en tant que « créateurs " par les médias. En 1987, Montana s'est vu décerner une récompense de la Fondation nationale des arts (National Endowment for the Arts). Dollis a reçu le même prix en 2011. En 1997, Harrison a reçu des mains du maire Marc Morial un prix d'excellence "pour l'ensemble de ses réalisations artistiques".

Labandon graduel du secret est une autre conséquence de la visibilité accrue des traditions afro-indiennes. Même si l'itinéraire suivi par les tribus le jour de Mardi Gras demeure encore inconnu, le nom des rues traversées par les Mardi Gras Indians lors des trois Super Sundays est en revanche divulgué à l'avance et le défilé se fait même sous escorte policière, à la demande (et à la charge) des organisateurs. De là à affirmer, comme le faisait récemment Cynthia Becker, que les Mardi Gras Indians ont été absorbés dans l'industrie touristique de la ville et qu'ils sont devenus « un bien de consommation ethnique qui symbolise La Nouvelle-Orléans », il n'y a qu'un pas ${ }^{70}$.

69 Sur le sens profond de cet exemple de "jazz diplomacy », lire King, C. Richard, "George Bush May Not Like Black People, But No One Gives a Damn About Indigenous Peoples : Visibility and Indianness After the Hurricanes ", American Indian Culture and Research Journal, vol. 32 n², 2008, pp. 35-42. Une série télévisée comme Tremé a, elle aussi, pris les Mardi Gras Indians comme illustration du paradigme de la résilience. Lire à ce sujet Godet, Aurélie, « 'How Many Mardi Gras Does One Man Need? Multiple Representations of Mardi Gras in Treme ", in HBO's Treme and Post-Katrina Catharsis : The Mediated Rebirth of a City, Gendrin, Dominique, Catherine Dessinges et Shearon Roberts, dir., Lanham, Lexington Books (à paraître en janvier 2017).

70 Becker, Cynthia, art. cit., p. 49. Michael White formule une conclusion similaire dans "New Orleans's African American Musical Traditions : The Spirit and Soul of a City », p. 87-106, in Seeking Higher Ground, Marable, Manning et Kristen Clarke, dir., New York, Palgrave Macmillan, 2008. Voir aussi Lockwood, Charles, «Playing with Flaming Arrows : Representing and Performing 
Nuançons néanmoins le propos : si la visibilité et la marchandisation des pratiques black Indian constituent bien deux défis de taille pour les tribus indiennes de La Nouvelle-Orléans aujourd'hui ${ }^{71}$, elles ne semblent menacer la tradition que sur le long terme. Les traditions carnavalesques noires, en tant qu'elles mettent en scène la violence des rapports sociaux et une identité noire conquérante, se fondent avant tout sur la marginalisation (réelle et perçue) de la classe populaire noire par l'élite blanche de la ville. Or en dépit des efforts accomplis par la municipalité depuis 2010, le sort des habitants des quartiers dont sont issus les Mardi Gras Indians est toujours aussi précaire. « La plupart des résidents noirs de La Nouvelle-Orléans ont l'impression d'avoir perdu le contrôle politique de leur ville ", expliquait Lance Hill, directeur du Southern Institute for Education and Research de l'université Tulane, en $2012^{72}$. De quoi nourrir pour longtemps encore la frustration créatrice de ces maçons, charpentiers, couvreurs, peintres en bâtiment qui forment l'essentiel des tribus indiennes de la ville, et empêcher par là-même toute « dépolitisation » durable de l'objet.

\section{Conclusion}

Il est tentant d'analyser les pratiques culturelles « mardigridiennes " (pour reprendre un terme forgé par Donald Harrison, $\mathrm{Sr}^{73}$ ) soit comme un hommage aux peuples autochtones de Louisiane, soit comme un rituel festif apolitique, ouvrant sur un « tiers espace " poétique, voire magico-religieux. Cette interprétation est encouragée par les Mardi Gras Indians eux-mêmes qui avouent souvent être "possédés " par l'esprit de leurs ancêtres et ne pas connaître l'origine exacte de leurs traditions ou le sens de leurs chants. Comme cet article a cependant tenté de le montrer, le détour par la culture indienne des Grandes Plaines est le fruit d'un contexte politique bien précis : celui de la ségrégation qui a forcé les Noirs de La Nouvelle-Orléans à dissimuler leurs pratiques festives sous un jour plus « acceptable» juridiquement et socialement. Les processions afro-indiennes, au-delà de leur évidente dimension esthétique, sont en réalité la manifestation

the Mardi Gras Indians Post-Katrina », mémoire de Master, Université de Californie à Santa Barbara, 2011.

71 Lire à ce sujet Robertson, Campbell, "Want to Use My Suit? Then Throw Me Something ", New York Times, 23 mars 2010. Disponible en ligne : http://www.nytimes.com/2010/03/24/ us/24orleans.html?_r=0. Consulté le 9 mars 2016.

72 Cité dans Rivlin, Gary, "Why New Orleans's Black Residents Are Still Underwater After Katrina ", New York Times Magazine, 18 août 2015. Disponible en ligne : http://www.nytimes. com/2015/08/23/magazine/why-new-orleans-black-residents-are-still-under-water-after-katrina. html. Consulté le 9 mars 2016. Voir aussi, sur la disparition de la classe moyenne noire depuis I'ouragan, Casselman, Ben, "Katrina Washed Away New Orleans's Black Middle Class ", FiveThirtyEight.com, 24 août 2015. Disponible en ligne : http://fivethirtyeight.com/features/katrinawashed-away-new-orleanss-black-middle-class/. Consulté le 9 mars 2016.

73 Cité dans Abrahams, Roger D., Nick Spitzer, John F. Szwed et Robert Farris Thompson, op. cit., p. 5. 
d'une fierté raciale qui n'est pas sans rappeler celle du mouvement Black Power, dans lequel on retrouve d'ailleurs la même légitimation de la violence (physique ou métaphorique) et le même effort de réappropriation de l'espace urbain ${ }^{74}$.

Selon certains chercheurs comme David Elliot Draper ou Charles Lockwood, la commercialisation des traditions black Indian depuis les années 1970, combinée au respect croissant qui leur est témoigné par la municipalité depuis 2011, serait en voie de dégrader la culture populaire noire de La Nouvelle-Orléans en sanctionnant sa commercialisation et sa muséification ${ }^{75}$. Mais là encore, la dépolitisation n'est qu'un masque. La résistance des quartiers ouvriers noirs se poursuit, même si l'acte participatif dont elles témoignent emprunte des voies non électorales, non partisanes ou gouvernementales. Les Mardi Gras Indians, par leurs croyances et leurs pratiques, continuent (consciemment ou non, épisodiquement ou non) de " faire de la politique autrement ». Le déguisement possède chez eux une signification historique, sociale et économique différente de celles que lui donnent les associations carnavalesques Rex, Comus, Bacchus ou Endymion, ainsi qu'en témoigne la préférence donnée au mot suit par rapport au mot costume et les récentes tentatives d'en transformer le statut juridique de simple article vestimentaire à celui d'« œeuvres d'art sculpturales » protégées par la droit d'auteur ${ }^{76}$. L'art de se grimer et de se déguiser n'est pas un jeu théâtral de l'individu isolé, et n'a pas pour cible principale le visiteur d'un jour. Les Black Indians expriment haut et fort, le jour de Mardi Gras et la nuit de la Saint-Joseph, le besoin de reconnaissance d'une partie oubliée de la population américaine. Autrement dit, le fait que leurs rituels festifs soient devenus des événements touristiques ne signifie nullement qu'ils ne jouent plus un rôle important dans la vie sociale, politique, psychologique et artistique de la communauté qui les a vus naître.

On le voit ici, l'approche de la question raciale sous l'angle festif (que l'on parle d'ailleurs des Mardi Gras Indians ou d'autres traditions festives noires comme le Zulu Social Aid and Pleasure Club sus-mentionné $e^{77}$, les second lines ${ }^{78}$,

74 Lire sur ce point Peniel E. Joseph, The Black Power Movement : Rethinking the Civil Rights-Black Power Era, Londres, Routledge, 2013, chapitres 1, 6 et 7.

75 À la Martinique ou en Guadeloupe, le qualificatif de " doudouiste » décrit précisément ce basculement d'une soi-disant " authenticité » vers des pratiques folkloriques plus ostentatoires et considérées comme "non légitimantes».

76 Lire à ce sujet le passionnant article de Mirzaian, Azita, "The Utility of Prettiness : Copyright Protection for Mardi Gras Indian Suits in the Era of the Useful Article Analysis », Journal of the Copyright Society U.S.A. 59, été 2012, p. 747-772.

77 Monje, Carlos, Carnival and Society: The Zulu Social Aid and Pleasure Club, 1909-1961, mémoire de Master, Harvard University, 2000.

78 Regis, Helen A., "Second Lines, Minstrelsy, and the Contested Landscapes of New Orleans Afro-Creole Festivals », Cultural Anthropology, vol. 14 n4, novembre 1999, p. 472-504. 
les Baby Dolls ${ }^{79}$ ou les groupes Skull and Bones ${ }^{80}$ ) permet d'avoir accès à un discours revendicatif assez peu visible car peu médiatisée ${ }^{81}$. Elle fait émerger, pour reprendre les termes de James $\mathrm{C}$. Scott, le «texte caché » des groupes dominés. Ce n'est pas là son seul intérêt. Elle permet également de mettre en exergue la dimension performative des identités raciales aux États-Unis (rappelons qu'avant de se traduire dans la loi, le binarisme racial s'était mis en scène lors du carnaval de 1873, par exemple ${ }^{82}$ ) et de suggérer la profonde imbrication des notions de " classe » et de " race " dans les pratiques culturelles populaires noires de La Nouvelle-Orléans. Car s'ils insistent sur le prestige et l'empowerment nés de leur inscription dans la vie communautaire, les Indiens de Mardi Gras mettent aussi l'accent sur l'aspect matériel de leurs traditions (prix des matériaux utilisés pour les costumes, montant de la cotisation réglée par les membres des tribus, etc.) et sur le savoir-faire artisanal nécessaire à la conception de leurs costumes.

En résumé, les rituels festifs des Mardi Gras Indians de La Nouvelle-Orléans sont à la fois les vecteurs d'un rêve communautaire déterritorialisé et un objet politique ancré dans un contexte géographique, historique et économique précis. Le jeu sémiotique sur lequel ils s'appuient, parce qu'il brouille les frontières raciales tout en célébrant une certaine idée de l'identité noire, les rend de ce fait particulièrement fascinants pour les spécialistes de littérature et des arts du spectacle, les historiens, les ethnologues, les sociologues, voire même les politistes.

$\square$

79 Tradition née dans les années 1910 à l'initiative de prostituées noires, les «Baby Dolls » défilent de concert avec les Mardi Gras Indians et revendiquent une féminité sensuelle voire provocante. Vaz, Kim Marie, The "Baby Dolls »: Breaking the Race and Gender Barriers of the New Orleans Mardi Gras Tradition, Baton Rouge, Louisiana State University Press, 2013.

80 Sorte de mementi mori ambulants similaires aux Guédé haïtiens (et notamment au Baron Samedi, dont ils reprennent d'ailleurs le costume noir orné d'un dessin de squelette), les Bones Men (et Women ) traversent à pied les quartiers afro-américains de La Nouvelle-Orléans afin de rappeler aux résidents la vanité des plaisirs terrestres. Wade, Leslie A., "Women Artists Recycling the Skull : New Bone Gang Traditions in Post-Katrina New Orleans », Western Folklore vol. 74, n¹, hiver 2015, p. 30-57.

81 Encore aujourd'hui, « les Black Indians ne représentent qu'une expression, une rumeur, une image dans les journaux » pendant la période du carnaval. Kinser, Samuel, op. cit., p. 175. Les ouvrages grand public les plus récents sur le carnaval de La Nouvelle-Orléans ne les mentionnent qu'en passant. Voir par exemple Laborde, Errol, Mardi Gras : Chronicles of the New Orleans Carnival, Gretna, Pelican Publishing Company, 2013 ; O’Neill, Rosary, op. cit.

82 Sur cet épisode aujourd'hui mieux connu de I'histoire du carnaval, lire Godet, Aurélie, " "The Missing Links to Darwin's Origin of Species" : darwinisme et racisme au carnaval de la NouvelleOrléans ", in Sexe, race et mixité dans l'aire anglophone, Michel Prum, dir., Paris, L'Harmattan, 2011, pp. 173-190. 


\section{Filmographie}

Katzmann, Lisa, Tootie's Last Suit, New York, Pomegranate Productions, 2006, 100 minutes. Martinez, Maurice, The Black Indians of New Orleans, USA, Chalmalma Media Institute, 1976, 33 minutes.

Osborn, Royce, All on a Mardi Gras Day, New Orleans, Spyboy Pictures, 2003, 155 minutes.

Rabaté, François, Carnaval à la Nouvelle-Orléans : le Mardi Gras mosaïque, Paris, Un Film à la patte, L'Envol et Arte, coll. "Carnavals », 2015, 52 minutes.

Simon, David et Eric Overmyer, Treme, USA, Home Box Office, 2010-2013.

Snedeker, Rebecca, By Invitation Only, Palmetto Pictures et New Day Films, 2006.

\section{Bibliographie}

"Super Sunday », MardiGrasNewOrleans.com. Disponible en ligne : http://www. mardigrasneworleans.com/supersunday.html. Consulté le 9 mars 2016.

Abrahams, Roger D., Nick Spitzer, John F. Szwed et Robert Farris Thompson, Blues for New Orleans : Mardi Gras and America's Creole Soul, Philadelphie : University of Pennsylvania Press, 2006.

Alvarez, Luis, The Power of the Zoot : Youth Culture and Resistance during World War II, Berkeley, University of California, 2008.

Anderson, Benedict, Imagined Communities : Reflections on the Origin and Spread of Nationalism, New York, Verso, 2006 [1983].

Arnaud, Lionel et Christine Guionnet, dir., Les Frontières du politique : enquêtes sur les processus de politisation et dépolitisation, Rennes, PUR, 2005.

Aubert, Guillaume, «The Blood of France : Race and Purity of Blood in the French Atlantic World », William and Mary Quarterly vol. 61 n³, 2004, p. 439-478.

Bakhtine, Mikhaïl, Le principe dialogique, Paris, Seuil, 1981.

Beauchez, Jérôme, L'empreinte du poing : la boxe, le gymnase et leurs hommes, Paris, Éditions de l'EHESS, 2014, coll. " Cas de figure».

Becker, Cynthia, « New Orleans Mardi Indians : Mediating Racial Politics from the Backstreets to Main Street», African Arts vol. 46, n², 2013, pp. 36-49.

Bellour, Hélène et Samuel Kinser, «Amerindian Masking in Trinidad's Carnival : The House of Black Elk in San Fernando ", in TDR: The Drama Review vol. 42 n³ (numéro spécial «Trinidad and Tobago Carnival » Coordonné par Milla Cozart Riggio), 1998, p. 147-169.

Bennett, James B., Religion and the Rise of Jim Crow in New Orleans, Princeton, Princeton University Press, 2016.

Berry, Jason, "Controversy Swirls Around Mardi Gras Indian Origins », New Orleans TimesPicayune, 17 février 1984, p. 6.

- Jonathan Foose et Tad Jones, Up From the Cradle of Jazz, Athens, University of Georgia Press, 1986.

Blumenfeld, Larry, "Beyond Jazzfest, Ruffled Feathers in New Orleans ", Village Voice, 22 juin 2011. Disponible en ligne : http://www.villagevoice.com/2011-06-22/music/beyondjazzfest-ruffled-feathers/2/. Consulté le 9 mars 2016.

Bragg, Rick, "Another Battle of New Orleans : Mardi Gras », New York Times, 19 février 1995. Disponible en ligne : http://www.nytimes.com/1995/02/19/us/another-battle-ofnew-orleans-mardi-gras.html?pagewanted=all. Consulté le 9 mars 2016.

Carrington, Ben, Race, Sport and Politics : The Sporting Black Diaspora, London, Sage, 2010. 
Casselman, Ben, " Katrina Washed Away New Orleans's Black Middle Class », FiveThirtyEight. com, 24 août 2015. Disponible en ligne : http://fivethirtyeight.com/features/katrina-washed-away-new-orleanss-black-middle-class/. Consulté le 9 mars 2016.

Clark, Emily, The Strange History of the American Quadroon : Free Women of Color in the Revolutionary Atlantic World, Chapel Hill, University of North Carolina Press, 2013.

Cohen, Abner, Masquerade Politics : Explorations in the Structure of Urban Cultural Movements, Berkeley, University of California Press, 1993.

Coulon, Christian, "La cuisine comme objet politique ", Revue internationale de politique comparée vol. 6 n², été 1999, p. 311-320.

Deloria, Philip J., Playing Indian, New Haven, Yale University Press, 1998.

Dessens, Nathalie, From Saint-Domingue to New Orleans : Migration and Influences, Gainesville, University Press of Florida, 2007.

—, " Cultures plurielles et hybridation : fêtes et célébrations à La Nouvelle-Orléans (18031840) ", in Dessens, Nathalie et Jean-Pierre Le Glaunec, Interculturalité : la Louisiane au carrefour des cultures, Presses Universitaires de Laval, 2016, p. 137-164.

DeVore, Donald E., Defying Jim Crow : African American Community Development and the Struggle for Racial Equality in New Orleans, 1900-1960, Baton Rouge, Louisiana State University, 2015.

Dominguez, Virginia, White By Definition : Social Classification in Creole Louisiana, New Brunswick, Rutgers University Press, 1986.

Draper, David Elliot, "The Mardi Gras Indians : The Ethnomusicology of Black Associations in New Orleans ", thèse de doctorat, Tulane University, 1973.

Dyson, Michael E., Come Hell or High Water : Hurricane Katrina and the Color of Disaster, New York, Basic Civitas, 2006.

Evans, Freddi Williams, Congo Square: African Roots in New Orleans, Lafayette, University of Louisiana at Lafayette Press, 2011.

Fensterstock, Alison, "Mardi Gras Indian Queens Stake Their Claim to an Essential Role in a Singular New Orleans Tradition », Times-Picayune, 2 mars 2014. Disponible en ligne: http://www.nola.com/mardigras/index.ssf/2014/03/mardi_gras_indian_queens_stake. html. Consulté le 9 mars 2016.

Fertel, Rien, Imagining the Creole City : The Rise of Literary Culture in Nineteenth-Century New Orleans, Baton Rouge, Louisiana State University Press, 2014.

Foucault, Michel, «Des espaces autres », in Dits et écrits, tome IV, Daniel Defert et François Ewald, dir., Paris, Gallimard, 1994, p. 752-762.

Gerhman, Mary, The Free People of Color of New Orleans : An Introduction, New Orleans, 1994.

Gill, James, Lords of Misrule: Mardi Gras and the Politics of Race in New Orleans, Jackson, University Press of Mississippi, 1997.

Godet, Aurélie, "'The Missing Links to Darwin's Origin of Species' : darwinisme et racisme au carnaval de la Nouvelle-Orléans ", in Sexe, race et mixité dans l'aire anglophone, Michel Prum, dir., Paris, L'Harmattan, 2011, pp. 173-190.

—, " 'How Many Mardi Gras Does One Man Need? Multiple Representations of Mardi Gras in Treme ", in Gendrin, Dominique, Catherine Dessinges et Shearon Roberts, dir., HBO's Treme and Post-Katrina Catharsis: The Mediated Rebirth of a City, Lanham : Lexington Books (à paraître en janvier 2017).

Gotham, Kevin Fox, « Marketing Mardi Gras : Commodification, Spectacle, and the Political Economy of Tourism in New Orleans », Urban Studies vol. 39 n¹0, 2002, p. 1735-1756. 
- Authentic New Orleans : Tourism, Culture and Race in the Big Easy, New York, New York University Press, 2007.

Gunbar, Susan, Racechanges : White Skin, Black Face in American Culture, New York, Oxford University Press, 1997.

Hanger, Kimberly S., Bounded Lives, Bounded Places : Free Black Society in Colonial New Orleans, 1769-1803, Durham, Duke University Press, 1997.

Hernandez, Julie, "Réécritures urbaines : héritages créoles et empreinte étasunienne à La Nouvelle-Orléans », RITA n¹, décembre 2008. Disponible en ligne : http://www.revuerita.com/dossier-thema-32/rritures-urbaines-thema-848.html. Consulté le 9 mars 2016.

Hirsch, Arnold, "Simply a Matter of Black and White : The Transformations of Race and Politics in Twentieth-Century New Orleans ", in Creole New Orleans : Race and Americanization, Hirsch, Arnold R. et Joseph Logdson, dir., Baton Rouge, Louisiana State University Press, 1992, p. 262-320.

Hirsch, Arnold R., «Fade To Black : Hurricane Katrina and the Disappearance of Creole New Orleans ", Journal of American History vol. 94 n³, 2007, p. 752-761.

Hobsbawm, Eric et Terence O. Ranger, The Invention of Tradition, Cambridge, Cambridge University Press, 1992.

Ingersoll, Thomas N., Mammon and Manon in Early New Orleans : The First Slave Society in the Deep Society, 1718-1819, Knoxville, University of Tennessee Press, 1999.

Jacobs, Claude et Andrew Kaslow, The Spiritual Churches of New Orleans : Origins, Beliefs, and Rituals of an African-American Religion, Knoxville, University of Tennessee Press, 1991.

Katz, William Loren, Black Indians : A Hidden Heritage, New York, Ethrac Publications, 1986.

Kelleher Schafer, Judith, Becoming Free, Remaining Free : Manumission and Enslavement in New Orleans, 1846-1862, Baton Rouge, Louisiana State University, 2003.

Kelley, Robin D. G., Race Rebels : Culture, Politics, and the Black Working Class, New York, Free Press, 1994.

King, C. Richard, "George Bush May Not Like Black People, But No One Gives a Damn About Indigenous Peoples: Visibility and Indianness After the Hurricanes ", American Indian Culture and Research Journal, vol. 32 n², 2008, pp. 35-42.

—, «Estrangements : Native American Mascots and Indian-Black Relations », in Confounding the Color Line : The Indian-Black Experience in North America, Brooks, James F., dir., Lincoln, University of Nebraska Press, 2002.

Kinser, Samuel, Carnival American Style : Mardi Gras at New Orleans and Mobile, Chicago, University of Chicago Press, 1990.

— "Violence Ritually Enjoined : The Mardi Gras Indians of New Orleans », Cahiers de littérature orale n³7, 1995, p. 115-149.

Laborde, Errol, Mardi Gras : Chronicles of the New Orleans Carnival, Gretna, Pelican Publishing Company, 2013.

Le Dantec-Lowry, Hélène, "Reading Women's Lives in Cookbooks and Other Culinary Writings : A Critical Essay », Revue française d'études américaines n¹16, 2008, p. 99-122.

Le Menestrel, Sara, La Voie des Cadiens : tourisme et identité en Louisiane, Paris, Belin, 1999.

— , "Créolisation, imaginaire racial et marché musical franco-louisianais », in Raulin, Anne et Susan Rogers, dir., Parallaxes transatlantiques : pour une anthropologie réciproque France-États-Unis, Paris, CNRS Éditions, 2012, p. 79-108.

- " "Live Music and Noise Debates in New Orleans Post-Katrina », Oxford Handbooks Online, novembre 2014. Disponible en ligne : http://www.oxfordhandbooks.com/ 
view/10.1093/oxfordhb/9780199935321.001.0001/oxfordhb-9780199935321-e115? print=pdf. Consulté le 9 mars 2016.

Lewis, Pierce, New Orleans : The Making of an Urban Landscape, Charlottesville, University of Virginia Press, 2003.

Lhamon, Jr., William T., Raising Cain. Blackface Performance from Jim Crow to Hip-Hop, Cambridge, Harvard University Press, 1998. Traduit en français par Sophie Renaut sous le titre Raising Cain : représentations du blackface de Jim Crow à Michael Jackson, éditions Kargo et l'Éclat, 2004.

Lipsitz, George, "Mardi Gras Indians : Carnival and Counter- Narrative in Black New Orleans », Cultural Critique n 10 (automne 1988), p. 99-121.

—, Time Passages : Collective Memory and American Popular Culture, Minneapolis, University of Minnesota Press, 2001 [1990].

Lockwood, Charles, « Playing with Flaming Arrows : Representing and Performing the Mardi Gras Indians Post-Katrina ", mémoire de Master, Université de Californie à Santa Barbara, 2011.

Lodgson, Joseph et Caryn Cossé Bell, "The Americanization of Black New Orleans », in Creole New Orleans : Race and Americanization, Hirsch, Arnold R. et Joseph Logdson, dir., Baton Rouge, Louisiana State University Press, 1992, p. 201-261.

Lovett, Laura, "'African and Cherokee by Choice' : Race and Resistance under Legalized Segregation ", in Confounding the Color Line: The Indian-Black Experience in North America, Brooks, James F., dir., Lincoln, University of Nebraska Press, 2002.

Maldonado, Charles, "The Mardi Gras Indians and the NOPD », Gambit, 14 février 2012. Disponible en ligne : http://www.bestofneworleans.com/gambit/the-mardi-gras-indiansand-the-nopd/Content?oid=1956440. Consulté le 9 mars 2016.

Marche, Guillaume, "Why Infrapolitics Matter ", Revue française d'études américaines $\mathrm{n}^{\circ} 131,2012$, p. 3-18.

Martin, Denis-Constant, Politics Behind the Mask : Studying Contemporary Carnivals in Political Perspective. Theoretical and Methodological Suggestions, Paris, CERI, "Questions de recherche », 2001. Disponible en ligne : www.ceri-sciences-po.org/ publica/qdr.htm. Consulté le 9 mars 2016.

— , Sur la piste des OPNI (objets politiques non identifiés), Paris, Karthala, 2002.

- Le Gospel afro-américain. Des spirituals au rap religieux, Paris, Cité de la musique / Actes Sud, 2008 [1998].

Massey, Douglas et Nancy Denton, American Apartheid. Segregation and the Making of the Underclass, Cambridge, Harvard University Press, 1998.

McFalls, Laurent, Julie Perreault, Nicolas Liorzou et Anca-Elena Mot, Construire le politique : contingence, causalité et connaissance dans la science politique contemporaine, Montréal, Presses de I'Université Laval, 2006.

Melucci, Alberto, Challenging Codes : Collective Action in the Information Age, Cambridge, Cambridge University Press, 1996.

Michna, Catherine, «Performance and Cross-Racial Storytelling in Post-Katrina New Orleans : Interviews with John O'Neal, Carol Bebelle, and Nicholas Slie »,TDR: The Drama Review, vol. 57 n¹, été 2013, pp. 48-69.

Mirzaian, Azita, "The Utility of Prettiness : Copyright Protection for Mardi Gras Indian Suits in the Era of the Useful Article Analysis ", Journal of the Copyright Society U.S.A. 59, été 2012, p. 747-772.

Mitchell, Reid, All On a Mardi Gras Day. Episodes in the History of New Orleans Carnival, Cambridge, Harvard University Press, 1999. 
Monje, Carlos, Carnival and Society: The Zulu Social Aid and Pleasure Club, 1909-1961, mémoire de Master, Harvard University, 2000.

Moreiras, Alberto, "Infrapolitical Literature. Hispanism and the Border », CR : The New Centennial Review vol. 10 n², 2010, p. 183-204.

Mulot, Stéphanie, "La trace des masques : identité guadeloupéenne entre pratique et discours ", Ethnologie française vol. 33 n¹, 2003, p. 111-122.

Nystrom, Justin, New Orleans after the Civil War : Race, Politics, and a New Birth of Freedom, Baltimore, Johns Hopkins University Press, 2010.

O'Neill, Rosary, New Orleans Carnival Krewes : The History, Spirit and Secrets of Mardi Gras, Charleston, The History Press, 2014.

Patterson, Orlando, "Four Modes of Ethno-Somatic Stratification : The Experience of Blacks in Europe and the Americas », in Ethnicity, Social Mobility, and Public Policy: Comparing the USA and UK, Glenn C. Loury, Tariq Modood, Steven M. Teles, dir., Cambridge, Cambridge University Press, 2005, p. 67-122.

Pearse, Andrew, "Carnival in Nineteenth Century Trinidad », Caribbean Quarterly vol. 4 n'3-4, 1956, p. 4-41.

Peniel E. Joseph, The Black Power Movement : Rethinking the Civil Rights-Black Power Era, Londres, Routledge, 2013.

Reckdahl, Katy, « New Orleans Police Handling of Mardi Gras Indian Events Draws Praise/ Criticism », Times-Picayune, 20 mars 2010. Disponible en ligne : http://www.nola.com/ crime/index.ssf/2010/03/new_orleans_police_handling_of.html. Consulté le 9 mars 2016.

— , «Decade After St. Joseph's Day Incident, Relations Between Police and Mardi Gras Indians Much Improved ", New Orleans Advocate, 18 mars 2015. Disponible en ligne: http://www.theneworleansadvocate.com/news/11880449-123/decade-after-st-josephsday. Consulté le 9 mars 2016.

- "Crackdown a Decade Ago Touched Off Powder Keg ", The New Orleans Advocate, 19 mars 2015. Disponible en ligne : http://www.theneworleansadvocate.com/ news/11880449-123/decade-after-st-josephs-day. Consulté le 9 mars 2016.

— , "St. Joseph's Night Gone Blue », Gambit, 29 mars 2005. Disponible en ligne : http:// www.bestofneworleans.com/gambit/st-josephs-night-gone-blue/Content?oid=1244049. Consulté le 9 mars 2016.

Regis, Helen A., "Second Lines, Minstrelsy, and the Contested Landscapes of New Orleans Afro-Creole Festivals », Cultural Anthropology, vol. 14 n4, novembre 1999, p. 472-504.

Roach, Joseph, Cities of the Dead : Circum-Atlantic Performance, New York, Columbia University Press, 1996.

Robertson, Campbell, "Want to Use My Suit ? Then Throw Me Something ", New York Times, 23 mars 2010. Disponible en ligne : http://www.nytimes.com/2010/03/24/us/24orleans.html?_r=0. Consulté le 9 mars 2016.

Rydin, Yvonne, "Justice and the Geography of Hurricane Katrina », Geoforum n³7, 2006. Saavedra, Martha, Les terrains politiques du football, Paris, Khartala, 2010.

Sakakeeny, Matt, "Mardi Gras Indians (ca. 1800s) », KnowLA, Encyclopedia of Louisiana. Disponible en ligne : http://www.knowla.org/entry/827/. Consulté le 9 mars 2016.

Schechner, Richard, Between Theater and Anthropology, Philadelphia, University of Pennsylvania Press, 1985.

Scott, James C., Weapons of the Weak : Everyday Forms of Peasant Resistance, New Haven, Yale University Press, 1985. 
- Domination and the Arts of Resistance. Hidden Transcripts, New Haven, Yale University Press, 1990. Traduit en français par Olivier Ruchet sous le titre La Domination ou les arts de la résistance. Fragments du discours subalterne, Paris, Éditions Amsterdam, 2008.

- Decoding Subaltern Politics. Ideology, Disguise, and Resistance in Agrarian Politics, London, Routledge, 2012.

- " Infrapolitics and Mobilizations : A Response by James C. Scott », Revue française d'études américaines n¹31, 2012, p. 112-117.

Simmons, LaKisha Michelle, Crescent City Girls : The Lives of Young Black Women in Segregated New Orleans, Chapel Hill, University of North Carolina Press, 2015.

Smith, Michael P., « Buffalo Bill and the Mardi Gras Indians », Louisiana Cultural Vistas vol. $3 \mathrm{n}^{\circ} 3$, automne 1992, p. 12-15

—, Mardi Gras Indians, Gretna, Pelican Publishing, 1993.

Solnit, Rebecca et Rebecca Snedeker, dir., Unfathomable City : A New Orleans Atlas, Berkeley, California University Press, 2013.

Souther, Souther, New Orleans on Parade : Tourism and the Transformation of the Crescent City, Baton Rouge, Louisiana State University Press, 2006.

—, "The Disneyfication of New Orleans : The French Quarter as Facade in a Divided City », Journal of American History vol. 94 n³, 2007, p. 804-811.

Sparks, Randy J. et Romain Huret, dir., Hurricane Katrina in Transatlantic Perspective, Baton Rouge, Louisiana State University, 2014.

Spear, Jennifer M., Race, Sex, and Social Order in Early New Orleans, Baltimore, Johns Hopkins University Press, 2009.

Thomas, Lynell L., Desire and Disaster in New Orleans : Tourism, Race, and Historical Memory, Durham, Duke University Press, 2014.

Tregle, Jr., Joseph, "Creoles and Americans ", in Creole New Orleans : Race and Americanization, Hirsch, Arnold R. et Joseph Logdson, dir., Baton Rouge, Louisiana State University Press, 1992, p. 131-185.

Vaz, Kim Marie, The "Baby Dolls »: Breaking the Race and Gender Barriers of the New Orleans Mardi Gras Tradition, Baton Rouge, Louisiana State University Press, 2013.

Vidal, Cécile, "Caribbean Louisiana : Church, Métissage, and the Language of Race in the Mississippi Colony during the French Period ", in Louisiana : Crossroads of the Atlantic World, Philadelphia, University of Pennsylvania Press, 2013, p. 125-146.

Voutat, Bernard, "La science politique ou le contournement de l'objet », in Espaces-Temps vol. 76-77, 2001, pp. 6-15. Numéro special coordonné par Sophie Duchesne et Florence Haegel.

Wagner, Jacob A., "Creole Urbanism : Searching for an Urban Future in the Flooded Streets of New Orleans », Space and Culture 9, 2006.

Watts, Lewis et Eric Porter, New Orleans Suite : Music and Culture in Transition, Berkeley, University of California Press, 2013.

Wehmeyer, Stephen C., «Feathered Footsteps : Mythologizing and Ritualizing Black Indian Processions in New Orleans", in Carnival Art, Culture and Politics : Performing Life, Crichlow, Michaeline, dir., Londres, Routledge, 2013, p. 29-47.

Weil, François, in Empires of the Imagination : Transatlantic Histories of the Louisiana Purchase, Kastor, Peter J. et François Weil, dir. Charlottesville, University of Virginia Press, 2009, p. 301-326.

Welburn, Ron, "Mardi Gras Indians : Spiritualism or Indian Stereotype? », in American Indians and Popular Culture, vol. 1 : "Media, Sports, and Politics », DeLaney Hoffman, Elizabeth, dir., Santa Barbara, ABC-CLIO, 2012, p. 311-328. 
Westphal, Bertrand, La géocritique : réel, fiction, espace, Paris, Minuit, 2007.

White, Michael, « New Orleans's African American Musical Traditions : The Spirit and Soul of a City », p. 87-106, in M. Marable et Kristen Clarke, Seeking Higher Ground: The Hurricane Katrina Crisis, Race and Public Policy Reader, New York, Palgrave Macmillan, 2008.

White, Richard et Patricia Nelson Limerick, The Frontier in American Culture, Berkeley, University of California Press, 1994.

White, Sophie, Wild Frenchmen and Frenchified Indians : Material Culture and Race in Colonial Louisiana, Philadelphie, University of Pennsylvania Press, 2012.

Williams, Karen L., Images of Uneasy Hybrids : Carnival and New Orleans, thèse de doctorat, Emory University, 1992.

Williams, Mary, «Private Lives and Public Orders : Regulating Sex, Marriage, and Legitimacy in Spanish Colonial Louisiana ", in Louisiana : Crossroads of the Atlantic World, Philadelphia, University of Pennsylvania Press, 2013, p. 147-164. 
